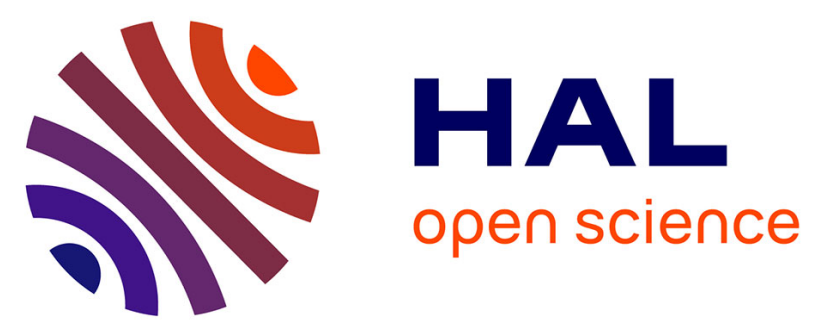

\title{
Quantification of Structure-Property Relationships for Plant Polyesters Reveals Suberin and Cutin Idiosyncrasies
}

Artur Bento, Carlos J.S. Moreira, Vanessa G. Correia, Rita Escórcio, Rúben Rodrigues, Ana S. Tomé, Nathalie Geneix, Johann J. Petit, Bénédicte Bakan, Christophe Rothan, et al.

\section{To cite this version:}

Artur Bento, Carlos J.S. Moreira, Vanessa G. Correia, Rita Escórcio, Rúben Rodrigues, et al.. Quantification of Structure-Property Relationships for Plant Polyesters Reveals Suberin and Cutin Idiosyncrasies. ACS Sustainable Chemistry \& Engineering, 2021, 9 (47), pp.15780-15792. 10.1021/acssuschemeng.1c04733 . hal-03435383

\section{HAL Id: hal-03435383 \\ https: / hal.inrae.fr/hal-03435383}

Submitted on 13 Dec 2021

HAL is a multi-disciplinary open access archive for the deposit and dissemination of scientific research documents, whether they are published or not. The documents may come from teaching and research institutions in France or abroad, or from public or private research centers.
L'archive ouverte pluridisciplinaire $\mathbf{H A L}$, est destinée au dépôt et à la diffusion de documents scientifiques de niveau recherche, publiés ou non, émanant des établissements d'enseignement et de recherche français ou étrangers, des laboratoires publics ou privés.

\section{(2)(1) $\$$}

Distributed under a Creative Commons Attribution - NonCommercial - NoDerivatives $\mid 4.0$ 


\title{
Quantification of Structure-Property Relationships for Plant Polyesters Reveals Suberin and Cutin Idiosyncrasies
}

\author{
Artur Bento, Carlos J. S. Moreira, Vanessa G. Correia, Rita Escórcio, Rúben Rodrigues, Ana S. Tomé, \\ Nathalie Geneix, Johann Petit, Bénédicte Bakan, Christophe Rothan, Oleksandr O. Mykhaylyk, \\ and Cristina Silva Pereira*
}

Cite This: ACS Sustainable Chem. Eng. 2021, 9, 15780-15792

Read Online

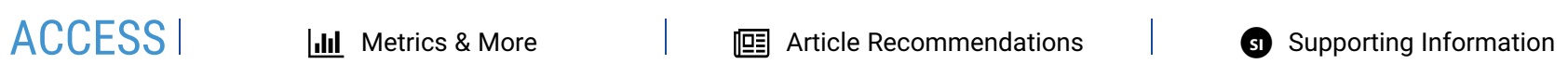

ABSTRACT: Polyesters, as they exist in planta, are promising materials with which to begin the development of "green" replacements. Cutin and suberin, polyesters found ubiquitously in plants, are prime candidates. Samples enriched for plant polyesters, and in which their native backbones were largely preserved, were studied to identify "natural" structural features; features that influence critical physical properties. Quantitative nuclear magnetic resonance (NMR), differential scanning calorimetry (DSC), and X-ray scattering methods were used to quantify structure-property relationships in these polymeric materials. The degree of esterification, namely, the presence of acylglycerol linkages in suberin and of secondary esters in cutin, and the existence of mid-chain epoxide groups defining the packing of the

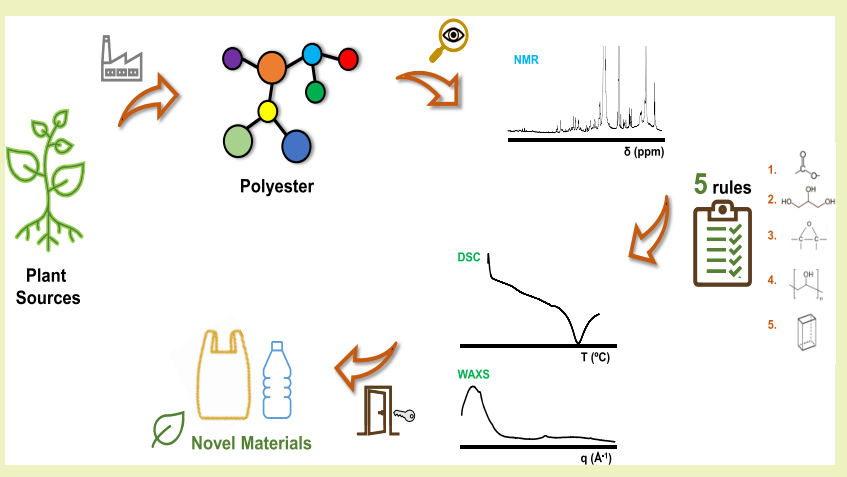
aliphatic chains were observed. This packing determines polymer crystallinity, the resulting crystal structure, and the melting temperature. To evaluate the strength of this rule, tomato cutin from the same genotype, studying wild-type plants and two wellcharacterized mutants, was analyzed. The results show that cutin's material properties are influenced by the amount of unbound aliphatic hydroxyl groups and by the length of the aliphatic chain. Collectively, the acquired data can be used as a tool to guide the selection of plant polyesters with precise structural features, and hence physicochemical properties.

KEYWORDS: plant industrial residues, ionic liquids, biopolymers, crystallinity, thermal resistance, quantitative NMR

\section{INTRODUCTION}

The use of synthetic plastics became widespread in the first half of the last century. This increased usage reflects their matchless durability, mechanical resistance, nonpermeability, versatility, as well as their low production costs. ${ }^{1}$ Plastics include a diverse range of materials, e.g., polyethylene, polyethylene terephthalate, polyvinyl chloride, and polystyrene, ${ }^{2}$ that are generated through harmful petroleum-based syntheses. These materials are employed in a vast range of products, many of which are single-use products. At the end of the product life cycle, the limited degradability of these materials contributes to a massive pollution problem and to the destruction of habitats around the world. The plague of plastic pollution, especially that of microplastics in marine ecosystems, has a staggering impact on wildlife. ${ }^{3}$ An increased awareness of this situation is driving the development of novel, sustainable alternatives as can be seen, for example, in the European Strategy for Plastics in a Circular Economy. ${ }^{4}$

During land colonization by plants, ca. 450 million years ago, plants acquired the capacity to synthesize cutin and, a related biopolymer, suberin. ${ }^{5}$ These cell-wall heteropolymers exhibit barrier properties and contribute to controlling water loss, providing insulation against climatic variability, and protecting against both abiotic and biotic pressures. ${ }^{5}$ They are ubiquitously present in land plants as a major component of the cell walls of the epidermis (cutin) and of periderms (suberin; e.g., tree bark and tuber periderms), where they build defensive hydrophobic barriers. $^{5}$ Naturally, they can be regarded as evolutionarily optimized, multifunctional biopolymers that control many aspects of plant biology. Changes to cutin and/or suberin result in many divergent phenotypes. Examples include alterations to: susceptibility to abiotic/biotic pressures, morphology, permeability, and others. ${ }^{6,7}$ These polymers (i.e., polyesters) constitute the third most abundant class of plant polymers, after cellulose/ hemicellulose and lignin. Thus, these materials should be

Received: July 13, 2021

Revised: September 29, 2021

Published: November 15, 2021 
considered as frontrunners in the development of bioplastics as alternatives to their synthetic counterparts.

Cutin and suberin are composed of an extremely complex heterogeneous mixture of monomeric units that contain both linear alkyl and aromatic moieties of monomers linked through aliphatic esters. ${ }^{8}$ Among the major differences are the presence of $\mathrm{C}_{16} / \mathrm{C}_{18} \omega$-hydroxyacids in cutin and the presence of both $\mathrm{C}_{16}-\mathrm{C}_{28} \omega$-hydroxyacids and $\mathrm{C}_{16}-\mathrm{C}_{26} \alpha, \omega$-diacids in suberin. ${ }^{9}$ Suberin extraction from plant periderms implies ester cleavage, which is typically achieved using nonspecific, hydrolysis-based processes that release monomeric constituents. ${ }^{10}$ Recovery of cutin usually involves time-consuming enzymatic-based hydrolyses, ${ }^{11}$ whereas subsequent chemical analyses often resort to extensive hydrolyses similar to those applied to suberin extraction. ${ }^{12}$ To date, extraction of suberin while preserving its esterified polymer network has only been achieved through the use of mild ionic-liquid catalysts that promote the selective and limited hydrolysis of acylglycerol esters. ${ }^{13}$ Recently, the suitability of this method for the isolation of suberin from cork $^{14}$ and potato peels ${ }^{15}$ has been demonstrated. This methodology allowed us to extract suberin while largely maintaining its native backbone, i.e., containing glycerol at levels close to its natural abundance. Additionally, a similar method could be used for the uncomplicated isolation of a highly esterified cutin matrix from tomato peels. This is due to the ability of the ionic liquid to solubilize the noncuticular polysaccharides and waxes. ${ }^{16}$ As a result, these ionic-liquidbased processes represent a unique approach with which to obtain materials enriched in plant polyesters that maintain a highly esterified polymeric backbone. The extracted materials should closely mimic the physicochemical properties of the polyester barriers found in planta. This strategy clearly differs from the design of polymer materials that require extensive depolymerization followed by subsequent repolymerization, ${ }^{10,17,18}$ yet we believe that these approaches should be seen as two complementary

With the aim of supporting knowledge-based development of materials derived from plant polyesters, the structures of distinct plant polyesters at both macro- and nanoscales were systematically characterized. Specifically, cork and potato peel were used as suberin sources and peel from tomato and red pepper were used as cutin sources. The chosen raw materials are readily available as agro-industrial residues and are generated in thousands of tons per year just in Europe. ${ }^{19}$ The plant polyesters were purified using established ionic liquid-based extraction methods. ${ }^{14,16}$ Structural and chemical characterization of the materials was performed by combining analyses using nuclear magnetic resonance (NMR) and wide-angle X-ray scattering (WAXS) complemented by thermal (differential scanning calorimetry, DSC) and morphological (scanning electron microscopy, SEM) analyses. To refine the understanding of how the chemical structure impacts the crystallinity and thermal resistance of the ensuing materials, cutin materials extracted from a defined tomato genotype (Micro-Tom) were also analyzed. Cutin was extracted from wild-type plants and also from two mutants defective in cutin biosynthesis. How each polymeric backbone, carrying distinct levels of esterification and mid-chain functionalities, assembles at both nano- and macrolevels, to form materials with diverse physicochemical properties, is discussed in great detail.

\section{MATERIAL AND METHODS}

Plant Material. Peels from commercially available tomato (Solanum lycopersicum) and red pepper (Capsicum annum) were manually removed, thoroughly washed, and then dried until constant weight at $60{ }^{\circ} \mathrm{C}$. After drying, the peels were milled using a Retsch ZM200 electric grinder (granulometry $0.5 \mathrm{~mm} ; 10000 \mathrm{rpm}$ ) and stored at room temperature until further processing. Micro-Tom cultivar tomatoes were provided by Institut National de la Recherche Agronomique (INRA) in Bordeaux; both mutants (gpat6 and cus1) were generated in the miniature cultivar Micro-Tom by an ethyl methanesulfonate (EMS) mutagenesis. ${ }^{20}$ All fruits used were in the red ripe developmental stage.

Granulated cork (bark from Quercus suber L.) was obtained from Amorim \& Irmãos SA (Santa Maria de Lamas, Portugal), and white potatoes (Solanum tuberosum L., cv. Monalisa) were from Batatas Mirense, Lda. (Mira, Portugal). Raw potato tubers were peeled; the peels were scraped in boiled water to ensure minimum pulp content and dried at $50^{\circ} \mathrm{C}$ until constant weight. Both plant sources were first milled (Retsch ZM200 electric grinder; granulometry $0.5 \mathrm{~mm} ; 10000 \mathrm{rpm}$ ); then, extractives were removed by sequential Soxhlet extraction with solvents of increasing polarity (dichloromethane, ethanol, and water) as previously described. ${ }^{13}$ The extractive-free preparations were washed in excess deionized water for complete removal of low-molecular-weight compounds and dried until constant weight at $60{ }^{\circ} \mathrm{C}$ before use.

Chemicals. Sodium hydroxide $(>98 \%)$ was purchased from José Manuel Gomes dos Santos. Methanol ( $\geq 99.8 \%)$, ethanol (99.8\%), dimethyl sulfoxide (DMSO, >99.99\%), hexane (>95\%), chloroform (>99.98\%), and dichloromethane (>99.99\%) were obtained from Fisher Chemical. Cholinium hydrogen carbonate $(\sim 80 \%$ in water), hexanoic acid $(>99.5 \%)$, sucrose monolaurate $(>97 \%)$, 4-octanol $(>97 \%)$, octanoic acid ( $\geq 98 \%)$, $p$-toluene-sulfonic acid monohydrate (>98\%), sulfuric acid (95-97\%), deuterated chloroform ( $\mathrm{CDCl}_{3}$, $99.8 \%)$, poly(1,4-butylene adipate $)\left(M_{\mathrm{w}} \sim 12000 \mathrm{~g} \cdot \mathrm{mol}^{-1}\right)$ were purchased from Sigma-Aldrich. Deuterated dimethyl sulfoxide (DMSO- $d_{6},>99.99 \%$ ) was obtained from Merck. Ethyl 4-hydroxy-3methoxy-cinnamate (99\%) was obtained from Alfa Aesar. Cholinium hexanoate was synthesized by dropwise addition of hexanoic acid to aqueous cholinium hydrogen carbonate in equimolar quantities, as previously described. ${ }^{21}$

Isolation of Plant Polyester-Enriched Materials. Suberin ${ }^{13}$ and cutin ${ }^{16}$ were extracted from cork/potato peel and tomato/pepper peels, respectively, as previously described. In brief, $2 \mathrm{~g}$ of the plant source (previously ground to a fine powder) was mixed in $20 \mathrm{~g}$ of ionic liquid (cholinium hexanoate) and incubated for $2 \mathrm{~h}$ at $100{ }^{\circ} \mathrm{C}$ without stirring. The reaction was stopped by the addition of $160 \mathrm{~mL}$ of DMSO. The insoluble residue was separated by filtration using a Nylon membrane filter $(0.45 \mu \mathrm{m})$. Suberin materials were recovered from the filtrate through precipitation in excess water, whereas cutin materials were recovered from the insoluble fraction retained on the filter. The ensuing materials, i.e., purified plant polyesters, were washed with an excess of deionized water with the aid of centrifugation (Beckman J2-MC centrifuge, $11655 \mathrm{~g}$ at $4{ }^{\circ} \mathrm{C}$ for $30 \mathrm{~min}$ ) and subsequently freeze-dried. The ensuing polyester powders were kept at room temperature until further analysis.

Synthesis of Octan-4-yl octanoate. A mixture of 4-octanol (1 $\mathrm{mL}$ ) with octanoic acid $(0.5 \mathrm{~mL})$ in the presence of a catalytic amount of $\mathrm{H}_{2} \mathrm{SO}_{4}$ and $p$-toluene-sulfonic acid monohydrate was prepared. The mixture was allowed to react at $60{ }^{\circ} \mathrm{C}$ under continuous stirring for 1 week. The end product was recovered by adding a saturated solution of $\mathrm{NaCl}$ to remove the excess 4-octanol (washing step). Yellow oil $(74.5 \%) ;{ }^{1} \mathrm{H} \mathrm{NMR}\left(400 \mathrm{MHz}, \mathrm{CDCl}_{3}\right): 4.88$ (quintet, $\left.J=5.4 \mathrm{~Hz}, 1 \mathrm{H}\right)$; $2.28(\mathrm{t}, J=7.5 \mathrm{~Hz}, 2 \mathrm{H}) ; 1.59(\mathrm{~m}, 3 \mathrm{H}) ; 1.49(\mathrm{~m}, 3 \mathrm{H}) ; 1.30(\mathrm{~m}, 19 \mathrm{H})$; $0.87(\mathrm{~m}, 14 \mathrm{H}) ;{ }^{13} \mathrm{C}$ NMR $\left(100 \mathrm{MHz}, \mathrm{CDCl}_{3}\right): 73.76 ; 36.34 ; 34.68$; $33.85 ; 31.87 ; 29.21 ; 27.64 ; 25.32 ; 22.76 ; 18.70 ; 14.14$.

Testing the Ability of an Ionic Liquid to Hydrolyze Ester Bonds Linking Distinct Monomers. Standard compounds carrying distinct types of esters bonds were tested, namely, octan-4-yl octanoate, sucrose monolaurate, ethyl 4-hydroxy-3-methoxy-cinnamate, and poly(1,4-butylene adipate). Either standard compound ( 50 mg) 
was mixed with cholinium hexanoate $(1: 10, \mathrm{w} / \mathrm{w})$ at $100{ }^{\circ} \mathrm{C}$, with stirring, for 2, 6, or $24 \mathrm{~h}$. To stop the poly(1,4-butylene adipate) reaction, an excess of water was added, then the reaction products were recovered by centrifugation $\left(15557 \mathrm{~g}, 4{ }^{\circ} \mathrm{C}, 30 \mathrm{~min}\right)$, lyophilized, and analyzed by NMR (see below). For the remaining standards, the reaction was stopped by placing the reaction mix on ice. The mixture was then acidified to $\mathrm{pH} 3 / 3.5$ with $1 \mathrm{M} \mathrm{HCl}$ solution, spiked with a known concentration of hexadecane (internal standard), and extracted three times using a dichloromethane/water partition. The dried combined organic phases were derivatized with $\mathrm{N}, \mathrm{O}$-bis(trimethylsilyl)trifluorocetamide in pyridine $(5: 1)$ for $30 \mathrm{~min}$ at 90 ${ }^{\circ} \mathrm{C}$. The TMS derivatives in the organic fractions were then analyzed by GC-MS (Agilent: 7820A GC and 5977B quadrupole MS; HP-5MS column) as follows: ramp temperature, $80^{\circ} \mathrm{C}$, then $4{ }^{\circ} \mathrm{C} \cdot \mathrm{min}^{-1}$ to 310 ${ }^{\circ} \mathrm{C}$ for $15 \mathrm{~min}$. The MS scan mode, with source at $230^{\circ} \mathrm{C}$ and electron impact ionization $\left(\mathrm{EI}^{+}, 70 \mathrm{eV}\right)$ was used for all samples. Each sample was analyzed in technical duplicate. Data acquisition was carried out using MSD ChemStation (Agilent Technologies); compounds were identified based on the equipment's spectral library (Wiley-National Institute of Standards and Technology).

Microscopic Analyses. Scanning electron microscopy (SEM; JEOL JSM-7001F microscope) was used to analyze the purified polyester samples.

Cryogenic Grinding Process. A RESTCH Cryomill equipped with a $25 \mathrm{~mL}$ grinding jar with 4 zirconium oxide grinding balls (10 $\mathrm{mm}$ ) was used. All samples of purified cutin were cryogenically milled at $-196^{\circ} \mathrm{C}$ (liquid nitrogen) using 200 milling cycles as follows: $3 \mathrm{~min}$ of precooling followed by nine milling cycles, each cycle consisting of 3 min of milling at $30 \mathrm{~Hz}$ followed by $0.5 \mathrm{~min}$ of intermediate cooling at 5 $\mathrm{Hz}$.

Nuclear Magnetic Resonance (NMR). NMR spectra of polyesters (either plant polyesters or the synthetic polyester) were recorded using an Avance III 800 CRYO (Bruker Biospin, Rheinstetten, Germany), except for the HSQC spectra of purified suberin, where an Avance II+ 800 was used instead (Bruker Biospin, Rheinstetten, Germany). All NMR spectra $\left({ }^{1} \mathrm{H},{ }^{1} \mathrm{H}-{ }^{1} \mathrm{H}\right.$ COSY, ${ }^{1} \mathrm{H}-{ }^{13} \mathrm{C}$ HSQC,${ }^{1} \mathrm{H}-{ }^{13} \mathrm{C}$ HMBC $)$ were acquired in DMSO- $d_{6}$ using $5 \mathrm{~mm}$ diameter NMR tubes at $60{ }^{\circ} \mathrm{C}$ as follows: $3 \mathrm{mg}$ of cryomilled cutin or $20 \mathrm{mg}$ of suberin in $400 \mu \mathrm{L}$ of DMSO- $d_{6}$. Quantitative ${ }^{31} \mathrm{P}$ NMR spectra of suberin and cutin were also recorded using an Avance III 500 (Bruker Biospin, Rheinstetten, Germany). ${ }^{22}$ NMR spectra of octan-4-yl octanoate were recorded using an Avance III 400 (Bruker Biospin, Rheinstetten, Germany). All NMR spectra $\left({ }^{1} \mathrm{H},{ }^{1} \mathrm{H}-{ }^{1} \mathrm{H}\right.$ COSY, ${ }^{1} \mathrm{H}-{ }^{13} \mathrm{C}$ HSQC, $\left.{ }^{1} \mathrm{H}-{ }^{13} \mathrm{C} \mathrm{HMBC}\right)$ were acquired in $\mathrm{CDCl}_{3}$ using $5 \mathrm{~mm}$ diameter NMR tubes at $25{ }^{\circ} \mathrm{C}$. MestReNova, Version 11.04-18998 (Mestrelab Research, S.L.) was used to process the raw data acquired in the Bruker spectrometers.

Differential Scanning Calorimetry (DSC). The DSC analyses were carried out in a TA Instruments Q200 calorimeter connected to a cooling system and calibrated with different standards (indium, empty cap). The polyester sample weights ranged from 9 to $11 \mathrm{mg}$. A temperature interval of -80 to $220^{\circ} \mathrm{C}$ has been studied, and the used heating/cooling rate was $10^{\circ} \mathrm{C} \cdot \mathrm{min}^{-1}$. To compare DSC and X-ray scattering results, enthalpy and melting points were measured using the first heating curve.

Wide-Angle X-ray Scattering (WAXS). WAXS data were collected using a laboratory SAXS/WAXS beamline (Xeuss 2.0, Xenocs, France) equipped with a liquid gallium MetalJet X-ray source (Excillum, Sweden) (wavelength $\lambda=1.34 \AA$ ), two sets of motorized scatterless slits for beam collimation, and a Pilatus 100k twodimensional (2D) pixel WAXS detector (Dectris, Switzerland). Loose cutin and suberin powder samples were enclosed between two flat kapton films and mounted on the beamline sample stage (the total sample thickness is about $1 \mathrm{~mm}$ ). 2D WAXS patterns were recorded in a transmission mode over a $q$ range of $1.3-3.5 \AA^{-1}$ [where $q=(4 \pi \sin \theta)$ / $\lambda=2 \pi / d$ is the length of the scattering vector, $\theta$ is one-half of the scattering angle, and $d$ is spacing in real space] using an exposure time of $1200 \mathrm{~s}$. The WAXS data were reduced (calibrated, integrated, and background-subtracted) using the Foxtrot software package supplied with the instrument.
Glycerol Quantification by High-Performance Liquid Chromatography (HPLC). The purified polyester samples were submitted to alkaline hydrolysis to release the hydrolyzable monomeric constituents. Briefly, the samples were treated with a solution of 0.5 $\mathrm{M} \mathrm{NaOH}$ in methanol/water $(1: 1, \mathrm{v} / \mathrm{v})$ at $95{ }^{\circ} \mathrm{C}$ for $4 \mathrm{~h}$. The mixture was cooled to room temperature, acidified to $\mathrm{pH} 3-3.5$ with $1 \mathrm{M} \mathrm{HCl}$, and extracted five times using a dichloromethane/water partition. The aqueous phases were analyzed by HPLC, using a Waters chromatographer Alliance 2695, connected to an LKB 2142 differential refractometer (Bromma, Sweden). Data acquisition was accomplished using the Empower 2 software (Waters). Chromatographic separation was undertaken at $60^{\circ} \mathrm{C}$ using an Aminex HPX-87H column $(300 \mathrm{~mm}$ $\times 7.8 \mathrm{~mm}$ ) with a $9 \mu \mathrm{m}$ particle size (Bio-Rad, Hercules, California). Elution was carried out isocratically, at a flow rate of $0.5 \mathrm{~mL} \cdot \mathrm{min}^{-1}$, with $0.005 \mathrm{~N} \mathrm{H}_{2} \mathrm{SO}_{4}$, and the sample volume injected was $20 \mu \mathrm{L}$. Glycerol quantification was carried out using an external calibration curve within the quantification limits of $0.1-1 \mathrm{mM}$. The samples were analyzed in triplicate in three independent experiments.

Semiquantitative Analyses of Crystalline Cellulose by DotBlot Immunoassay. The cryogenically milled cutin samples $(4 \mathrm{mg})$ were suspended in $1 \mathrm{~mL}$ of $3 \mathrm{M} \mathrm{NaOH}$. The suspension was vortexed for $1 \mathrm{~h}$, sonicated twice for 1 min each using a microtip (Q-Sonica). followed by $24 \mathrm{~h}$ under magnetic stirring. Standard crystalline cellulose (Avicel*PH-101, Sigma, France) was used for the quantification of crystalline cellulose content in cutin samples. Dilution of both samples and standards was spotted in triplicate onto poly(vinylidene difluoride) (PVDF) blotting membrane (Amersham Hybond P $0.45 \mu \mathrm{m}, \mathrm{GE}$ Healthcare, France) with $2 \mu \mathrm{L}$ volume per dot. For the calibration curve, standards were spotted in decreasing concentrations: 0.8, 0.6, $0.4,0.2,0.1,0.08,0.06,0.04,0.02$, and $0.01 \mathrm{mg} \cdot \mathrm{mL}^{-1}$. The membrane was immersed in a $3 \%$ bovine serum albumin (BSA, Sigma, France) solution in phosphate-buffered saline (PBS $=137 \mathrm{mmol} \cdot \mathrm{L}^{-1} \mathrm{NaCl}, 2.7$ $\mathrm{mmol} \cdot \mathrm{L}^{-1} \mathrm{KCl}, 10 \mathrm{mmol} \cdot \mathrm{L}^{-1} \mathrm{Na}_{2} \mathrm{HPO}_{4}$, and $1.8 \mathrm{mmol} \cdot \mathrm{L}^{-1} \mathrm{KH}_{2} \mathrm{PO}_{4}$, $\mathrm{pH}$ 7.4) for $30 \mathrm{~min}$ and was washed three times with PBS-Tween (PBS with $0.05 \%$ Tween 20 ). The membrane was immersed in $1 / 1300$ diluted CMB3a recombinant protein ${ }^{23}$ in PBS-BSA-Tween (0.3\% BSA in PBS-Tween), followed by $120 \mathrm{~min}$ incubation, and finally washed with PBS-Tween as described above. The histidine tag of CMB3a was detected using an IgG anti-histidine mouse antibody (Sigma, France) diluted $1 / 2000$ in PBS-BSA-Tween and revealed by a peroxidase assay (Sigma, France) with the chemiluminescent substrate (Western Bright Quantum chemiluminescence HRP substrate, ADVANSTA-K12042D20, Menlo Park) and a Fuji Las3000 (Fujifilm, France) camera for detection. Image analysis was conducted with ImageJ (imagej.nih.gov/ $\mathrm{ij} /$ download.html). Each sample was analyzed in three independent assays; the obtained values reveal, semiquantitatively, the abundance of crystalline cellulose in the different samples.

Quantitative Analyses of Total Carbohydrate Content. To evaluate the sugar moiety content of the plant polyesters purified using the ionic-liquid-based processes, the samples were subjected to acid hydrolysis $\left(1 \mathrm{M} \mathrm{H}_{2} \mathrm{SO}_{4}\right.$ in methanol) for $4 \mathrm{~h}$ at $90^{\circ} \mathrm{C}$. The hydrolyzable sugars were recovered in the supernatant through centrifugation (18 514g, $4{ }^{\circ} \mathrm{C}, 20 \mathrm{~min}$ ), and the $\mathrm{pH}$ was neutralized using $5 \mathrm{M} \mathrm{NaOH}$ in water. All samples were dried under a flux of nitrogen at room temperature. Quantification of carbohydrates in the dried hydrolysates was performed using the total carbohydrate assay kit from SigmaAldrich according to the manufacturer's instructions. The samples were analyzed in triplicate in two independent experiments.

\section{RESULTS AND DISCUSSION}

Morphological Characterization Reveals Different Levels of Structural Organization. Cutin and suberin were purified from the corresponding plant sources using cholinium hexanoate $(2 \mathrm{~h})$ as previously established. ${ }^{14,16}$ Importantly, although these processes rely on the same catalyst, cholinium hexanoate, cutin is isolated from the cuticle as an insoluble component (following solubilization of polysaccharides and waxes in the ionic liquid), whereas suberin is recovered from the supernatant, which contains the ionic liquid as a soluble 
a
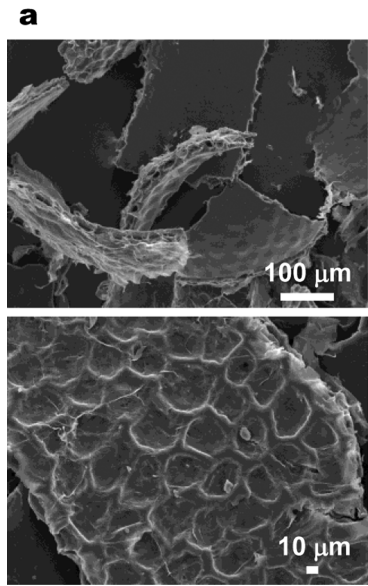
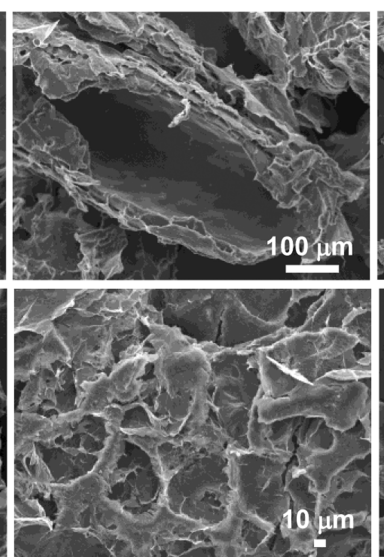

c
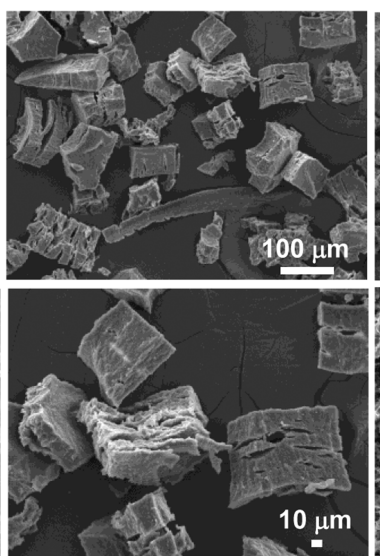

d

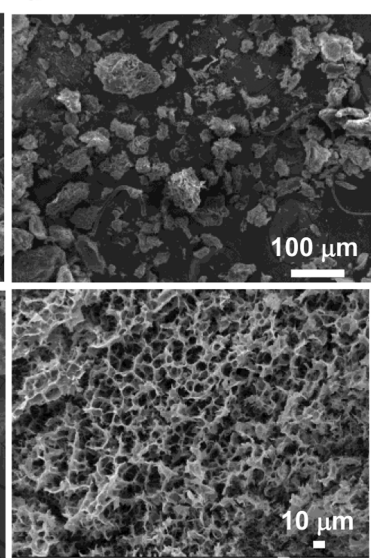

Figure 1. Morphological characterization of plant polyesters. SEM micrographs of cutin recovered from tomato peel (a) and pepper peel (b), and those of suberin from cork (c) and potato peel (d), following cholinium hexanoate extraction for $2 \mathrm{~h}$.

component, by precipitation in an excess of water (Figure S1). Naturally, the cutin and suberin samples exhibited very different morphological features, as observed by SEM (Figure 1). The integrity of the cellular structure was largely preserved following the ionic liquid-based extraction of cutin from either tomato or pepper peels. A clean, thick cutin-continuum that showed the epidermal cell grooves was revealed (Figure 1a,b). The purified cork and potato suberin samples consist of ordered and elongated polygon structures (Figure 1c) and of porous and irregular particles (Figure 1d), respectively. As such, the observed morphology of each type of polyester, upon extraction with cholinium hexanoate, is consistent with that previously reported for comparable samples, ${ }^{14,16}$ highlighting the reproducibility of the extraction method.

Ionic-Liquid-Mediated Hydrolysis is Ester-Type Specific. The capacity of an ionic liquid to hydrolyze octan-4-yl octanoate (secondary aliphatic ester), sucrose monolaurate (ester link between a fatty acid and a sugar), ethyl 4-hydroxy-3methoxy-cinnamate (ester link between an alcohol and an aromatic acid), and poly(1,4-butylene adipate) (synthetic linear polyester) was evaluated. This dataset aims to complement the data obtained previously with octyl-octanoate (primary aliphatic ester) and glyceryl-trioctanoate (acylglycerol ester) that showed virtually no ester cleavage and mild, yet progressive ester cleavage, respectively. ${ }^{13,16}$ All of the compounds tested here were largely resistant to the ionic-liquid-mediated hydrolysis achieving only $c a$. $5 \%$ of ester cleavage after $2 \mathrm{~h}$ (Figures S2 and S3). Analyses of longer reaction times (until $24 \mathrm{~h}$ ) demonstrated that the hydrolysis is ester-type-specific as follows: acylglycerol ester $>$ sugar ester $\cong$ aromatic ester $>$ secondary aliphatic ester $\cong$ primary aliphatic ester. Hydrolysis of poly(1,4-butylene adipate) (Figure S4) did not occur, similar to that observed before with octyl-octanoate. ${ }^{16}$ Collectively, the data showed that after $2 \mathrm{~h}$, the ionic-liquid-mediated mild cleavage of distinct ester types, except for primary aliphatic esters that remain intact, is consistent with the recovery of highly preserved esterified plant polyesters.

Nuclear Magnetic Resonance Unveils Quantitative Chemical Fingerprints for Each Plant Polyester. The elemental composition of either plant polyester under study is similar to that previously reported (Table S1). ${ }^{13,24-28}$ Note that small differences between the two polyester types, cutin and suberin, were observed. Small differences were also observed for same polyester type originating from different plant sources. In planta, the polyester backbone is linked to cell-wall polysaccharides, a hypothesis supported by experimental data. ${ }^{29-31}$ To test for the presence of polysaccharide moieties, the amount of hydrolyzable carbohydrates present in each polyester type was analyzed (Table S2). The amount of carbohydrates present in the polyester samples was below $0.1 \%$, with potato suberin reporting the highest value $\left(1.16 \mathrm{ng} \cdot \mathrm{mg}^{-1}\right)$ and pepper cutin reporting the lowest value $\left(0.12 \mathrm{ng} \cdot \mathrm{mg}^{-1}\right)$.

In recent studies, following successful solubilization of tomato cutin and cork suberin in DMSO, high-resolution, solution-state NMR spectra were acquired. ${ }^{14,16}$ These previous studies enabled more details of the chemistry of the two polymers (e.g., for tomato cutin, the esterification level, assignment of esters, free acids, and alcohols; ${ }^{16}$ for suberin, the ratio of linear aliphatic/acylglycerol esters and the relative abundance of acylglycerol configurations ${ }^{14}$ ). In the present study, this methodological approach was used by resorting to a cryoprobe to further enhance the sensitivity of the NMR analyses. Thus, it was possible to expand the list of assigned signals and to quantify specific molecular features. The ${ }^{1} \mathrm{H}$ NMR spectra for all samples were obtained at a high resolution and, in agreement with previous reports, exhibited many overlapping signals (Figure $2 \mathrm{aI}-\mathrm{dI}) .{ }^{14,16}$ The assignment of signals was achieved through a combination of $2 \mathrm{D}$ spectra $\left({ }^{1} \mathrm{H}-{ }^{1} \mathrm{H}\right.$ COSY, ${ }^{1} \mathrm{H}-{ }^{13} \mathrm{C}$ HSQC, and ${ }^{1} \mathrm{H}-{ }^{13} \mathrm{C} \mathrm{HMBC}$, Figures $\mathrm{S} 5-\mathrm{S} 16$, full list of assignments in Tables S3 and S4), guided by previous NMR data for tomato cutin $^{16}$ and cork suberin, ${ }^{14}$ as well as NMR data from oligomeric structures obtained through methanolysis of tomato peels. ${ }^{12}$

The analysis of the HSQC spectra for both cutin samples shows the presence of two aliphatic regions with the assignment of $\mathrm{CH}_{2}$ and $\mathrm{CH}_{3}$ groups from the aliphatic chains, the ester bonds, and the free mid-chain hydroxyl groups (Figure 2aII,bII). It was possible to assign two new signals related to secondary aliphatic ester bonds (SAE) and epoxide rings (Figure 2aIII,bIII). The secondary ester bonds were assigned to a ${ }^{13} \mathrm{C}$ shift of $72.61 \mathrm{ppm}$ and ${ }^{1} \mathrm{H}$ shift of $4.77 \mathrm{ppm}$, while the signal assigned to the epoxide rings (only detected in pepper cutin) has $a^{13} \mathrm{C}$ shift of $55.43 \mathrm{ppm}$ and a ${ }^{1} \mathrm{H}$ shift of $2.82 \mathrm{ppm}$. Moreover, in the HSQC spectrum of tomato cutin, it was only possible to detect secondary free hydroxyl groups ( $\mathrm{CHOH}$ mid-chain), which contrasts with the detection of signals related to both primary $\left(\mathrm{CH}_{2} \mathrm{OH}\right)$ and secondary hydroxyl $(\mathrm{CH}-(\mathrm{OH})-)$ groups in pepper cutin. 

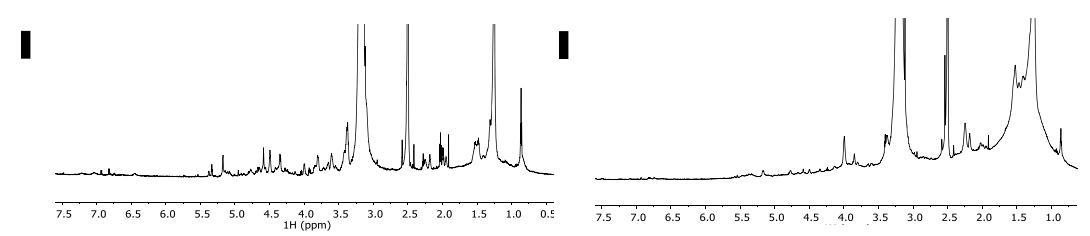

II
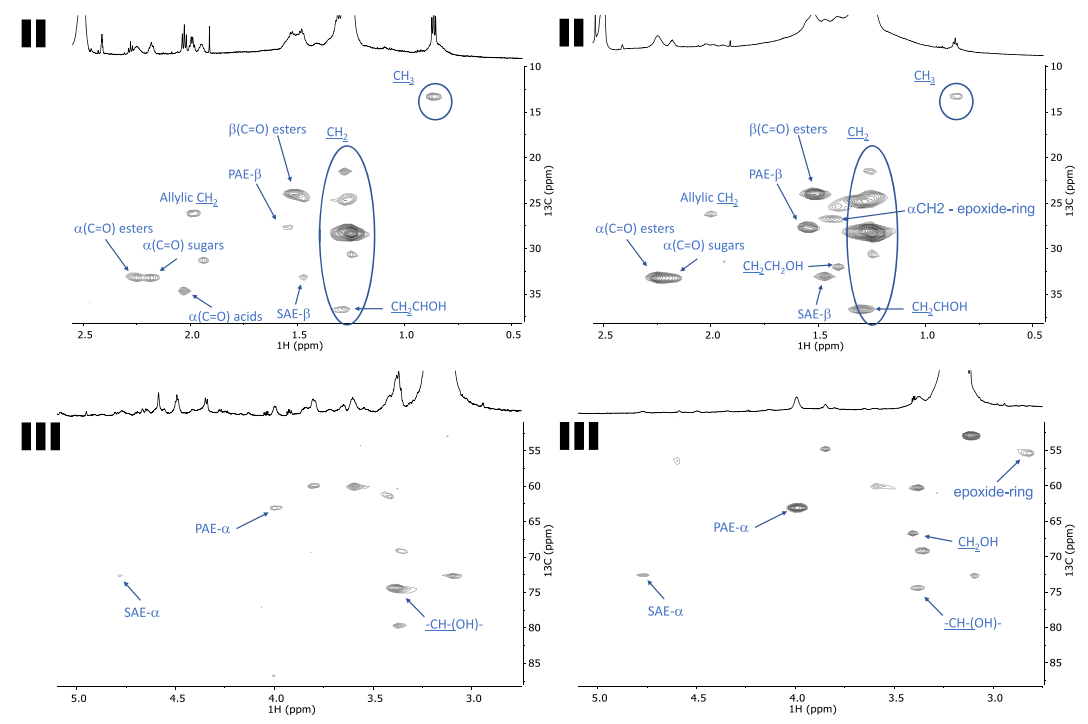

Cork Suberin

\section{Potato Suberin}
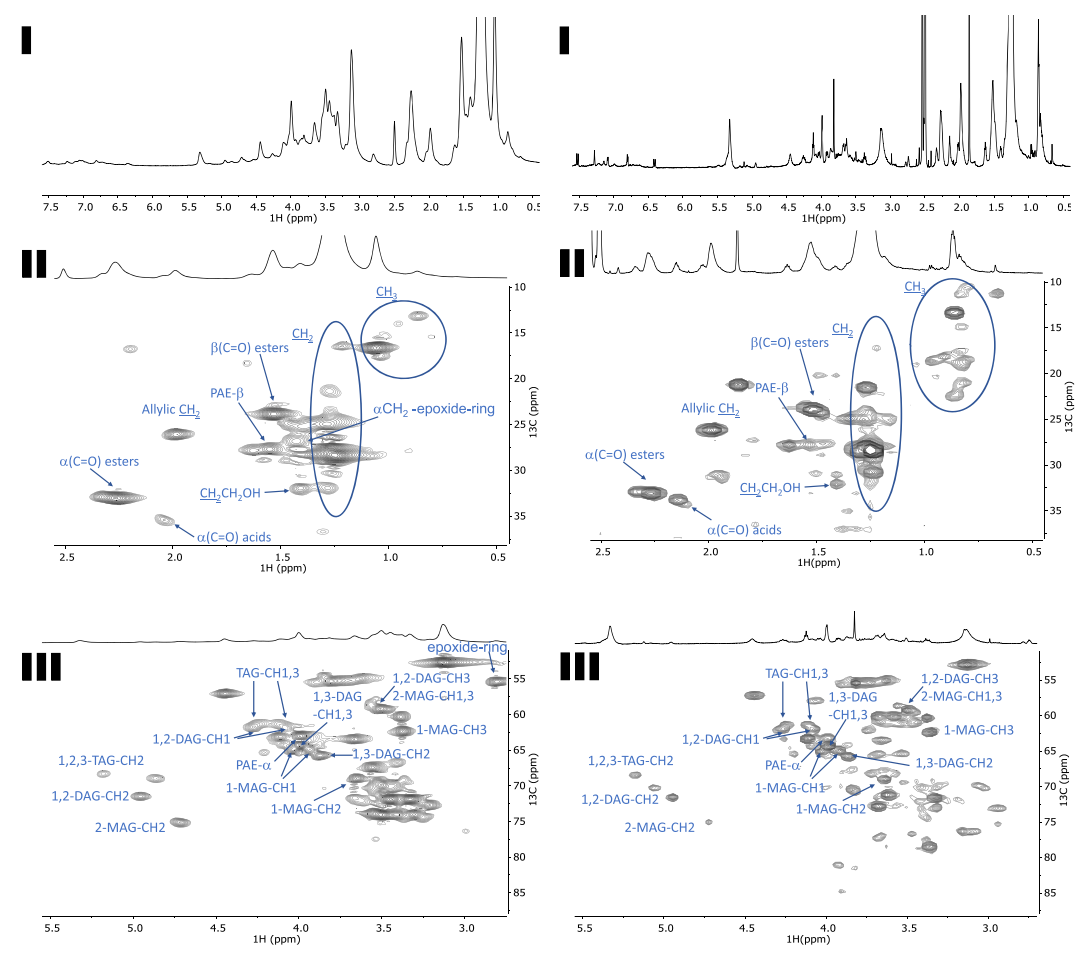

Figure 2. Wide-ranging NMR spectral characterization of tomato cutin (a); pepper cutin (b); cork suberin (c); and potato suberin (d) upon isolation using cholinium hexanoate extraction for $2 \mathrm{~h} .{ }^{1} \mathrm{H}$ NMR spectra of all samples (I) and the HSQC regions corresponding to aliphatics (II) and CH/CH2$\mathrm{X}$ aliphatics (III). Some correlations (unlabeled) are uncertain or unidentified.

In the HSQC spectra of both cutin samples, two signals were detected for the $\alpha-(\mathrm{C}=\mathrm{O})$ with a ${ }^{13} \mathrm{C}$ shift of $33 \mathrm{ppm}$ associated with ${ }^{1} \mathrm{H}$ shifts of 2.25 and $2.19 \mathrm{ppm}$, which can be assigned to esters (Figure 2aII,bII). Vestigial amounts of microcrystalline 
Table 1. Chemical Characterization of Cutin and Suberin Samples ${ }^{a}$

\begin{tabular}{|c|c|c|c|c|c|c|}
\hline & & & \multicolumn{4}{|c|}{ purified plant polyesters } \\
\hline & & & \multicolumn{2}{|c|}{ cutin } & \multicolumn{2}{|c|}{ suberin } \\
\hline & & & tomato & pepper & cork & potato \\
\hline \multirow[t]{13}{*}{ NMR } & primary esters (\%) & & $20^{b}[0.2]$ & $59^{b}[1.77]$ & $30^{d}$ & $17^{d}$ \\
\hline & secondary esters (\%) & & $80^{b}[0.8]$ & $41^{b}[1.23]$ & & \\
\hline & total esters & & $1^{c}$ & $3^{c}$ & & \\
\hline & acylglycerol esters (\%) & & & & $70^{d}$ & $83^{d}$ \\
\hline & free $\operatorname{acids}^{e}(\%)$ & & 0.04 & n.d. & 0.47 & 0.66 \\
\hline & glycerol configuration $^{f}(\%)$ & $1,2,3-\mathrm{TAG}$ & n.d. & n.d. & 6 & 12 \\
\hline & & $1,2-\mathrm{DAG}$ & n.d. & n.d. & 19 & 18 \\
\hline & & 1,3-DAG & n.d. & n.d. & 25 & 38 \\
\hline & & 1-MAG & n.d. & n.d. & 23 & 28 \\
\hline & & 2-MAG & n.d. & n.d. & 27 & 4 \\
\hline & ${ }^{31} \mathrm{P}$ NMR quantification $\left(\mathrm{mmol} \cdot \mathrm{g}^{-1}\right)$ & free acids & 0.07 & 0.05 & 0.30 & 0.43 \\
\hline & & $\mathrm{OH}$ aliphatics & 4.17 & 0.34 & 2.39 & 5.89 \\
\hline & & $\mathrm{OH}$ aromatics & n.d. & 0.01 & 0.44 & 0.49 \\
\hline HPLC & glycerol (\%, w/w) & & n.d. & n.d. & $3.86 \pm 0.31$ & $2.16 \pm 0.20$ \\
\hline
\end{tabular}

${ }^{a}$ NMR quantification of the main functional groups in both plant polyesters from each source and HPLC quantification of glycerol present in the recovered polymeric materials. ${ }^{b}$ Relative abundances of primary and secondary esters present in cutin calculated through the integration of signals in the ${ }^{1} \mathrm{H}$ NMR spectra. Values in brackets correspond to the normalized values using the amount of the total esters in each sample. ${ }^{c}$ Integration of the total ester signal was normalized to benzene for comparison between samples. ${ }^{d}$ Relative abundances of linear and acylglycerol esters present in suberin calculated by the integration of each signal's contour volume in the ${ }^{1} \mathrm{H}$ and ${ }^{13} \mathrm{C}$ HSQC spectra. ${ }^{e}$ Content of free acids relative to the total

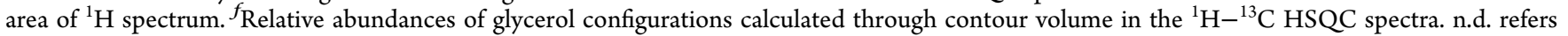
to not detected. TAG, DAG, and MAG stand for triacylglycerol, diacylglycerol, and monoacylglycerol, respectively.

cellulose were previously detected in tomato cutin samples purified by cholinium hexanoate. ${ }^{16}$ Accordingly, the ${ }^{1} \mathrm{H}$ shift at $2.19 \mathrm{ppm}$ may be associated with the presence of cellulose esters. The ${ }^{13} \mathrm{C}$ shift at $34.66 \mathrm{ppm}$ and the ${ }^{1} \mathrm{H}$ shift at $2.03 \mathrm{ppm}$ were assigned to the free acids, a signal only present in tomato cutin and absent in pepper cutin (Figure 2aII,bII). In the HMBC spectrum of these biopolyesters (Figures S7 and S10), ${ }^{3} J_{\mathrm{CH}}$ (three bond distance $\mathrm{C} \cdots \mathrm{H}$ ) couplings detected, involving the ester carbonyl quaternary carbons, provided additional support for the presence of signals derived from esters and acids. Two different quaternary carbonyl carbons were present, with small differences in their chemical shifts: the carbon in the primary aliphatic ester (PAE), at $172.34 \mathrm{ppm}$, and the carbon in the free acid, at $173.96 \mathrm{ppm}$. These assignments were confirmed by their ${ }^{3} J_{\mathrm{CH}}$ connectivity: the primary ester carbon was coupled with the methylene protons, and the free acid was not coupled with any proton group with a ${ }^{3} \mathrm{~J}_{\mathrm{CH}}$ coupling.

For both suberin samples, the HSQC spectra show the presence of two aliphatic regions with the assignment of $\mathrm{CH}_{2}$ and $\mathrm{CH}_{3}$ groups from the aliphatic chains, the ester bonds and the free primary hydroxyl groups (Figure 2cII,dII). In these two polymers, signals that could be assigned to all five glycerol configurations (1,2,3-triacylglycerol (TAG), 1,2-diacylglycerol (1,2-DAG), 1,3,-dyacylglycerol (1,3-DAG), 2-monoacylglycerol (2-MAG), and 1-monoacylglycerol (1-MAG)) were detected, as previously reported for cork suberin, ${ }^{14}$ and potato suberin. ${ }^{15}$ Moreover, the presence of the signal assigned to the epoxide groups was only observed in cork suberin at a ${ }^{13} \mathrm{C}$ shift of 55.41 ppm and a ${ }^{1} \mathrm{H}$ shift of $2.80 \mathrm{ppm}$, consistent with a previous report. $^{14}$

In both suberin samples, signals related to the presence of $\alpha$ $(\mathrm{C}=\mathrm{O})$ esters with a ${ }^{13} \mathrm{C}$ shift of $33.05 \mathrm{ppm}$ and a ${ }^{1} \mathrm{H}$ shift of $2.26 \mathrm{ppm}$, and the presence of free acids at a ${ }^{13} \mathrm{C}$ shift of 35.43 ppm and a ${ }^{1} \mathrm{H}$ shift of $2.03 \mathrm{ppm}$ were detected (Figure 2cII,dII). These assignments were confirmed by HMBC (Figures S13 and S16). Two different quaternary carbonyl carbons were observed: in the primary aliphatic ester, at $173.01 \mathrm{ppm}$, and in the free acid, at $175.63 \mathrm{ppm}$.

The ionic liquid-based approach extracts plant polyesters that retain high levels of esterification; this can be shown quantitatively through NMR. To infer the degree of esterification, the relative abundances of total aliphatic esters were estimated, through the integration of the corresponding NMR signals. This approach allowed us to discriminate the contributions of primary and secondary aliphatic esters in cutin, as well as primary and acylglycerol aliphatic esters in suberin (Table 1).

The ratio between primary and secondary esters provides meaningful information on the polymeric arrangement of cutin. Specifically, tomato cutin is enriched in secondary esters, whereas pepper cutin shows higher levels of primary esters. The amount of total esters in pepper cutin is 3-fold higher than that found in tomato cutin. This is consistent with the presence of a much more esterified cutin in pepper. Additionally, tomato cutin exhibits higher levels of free hydroxyl groups, which also supports the idea of a lower degree of esterification. ${ }^{32}$

The presence of free acids was observed in the ${ }^{1} \mathrm{H}$ and HSQC spectra from tomato cutin $(0.04 \%$, Table 1 and as reported previously $\left.{ }^{16}\right)$ but were undetected in the pepper cutin spectra. Glycerol was not detected in the HSQC analyses of either cutin sample (Figure 2aIII,bIII), nor could it be quantified by liquid chromatography (i.e., below the quantification limit of the method), which is consistent with a very low abundance of this monomer. $^{33}$

In contrast to cutin, glycerol is known to be a structural monomer in the polymeric arrangement of suberin. ${ }^{34}$ Resorting 


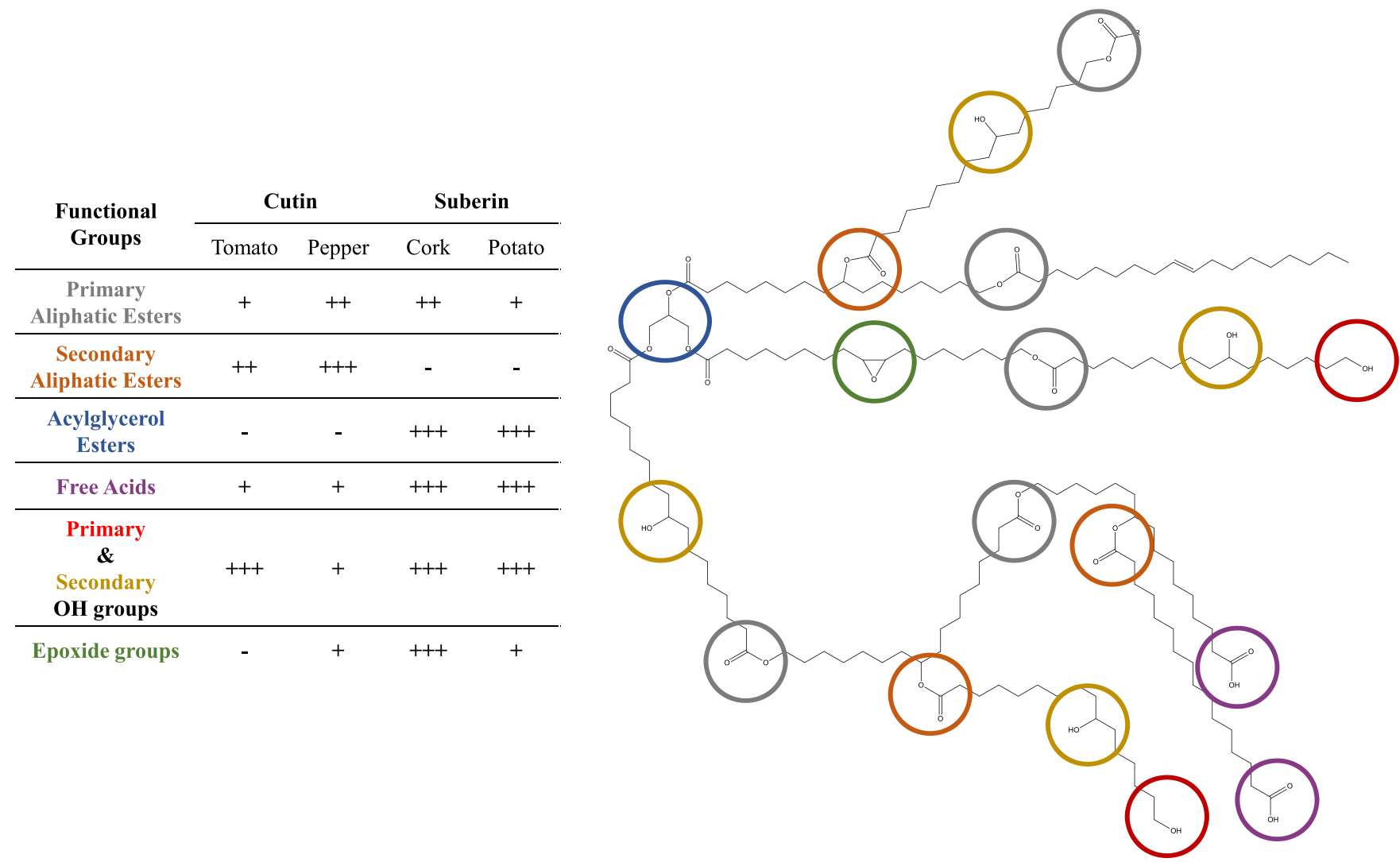

Figure 3. Schematic representation of the main mid-chain and end-chain functionalities (highlighted in different colors) of the plant polyesters cutin and suberin. Their relative abundance $(-$, absent; + , present) in different polyester types and from different sources is depicted to ease the understanding of the primary polymeric structure.

to liquid chromatography, the glycerol content found in cork and potato suberin was quantified, consisting of 3.86 and $2.16 \%$ $(\mathrm{w} / \mathrm{w})$, respectively. This difference suggests that cork suberin is more esterified than potato suberin. Bearing in mind the mode of action of cholinium hexanoate, a selective catalyst that mildly hydrolyzes the acylglycerol esters in suberin, the relative abundance of each acylglycerol configuration was inferred through their contour volume integrals in the HSQC spectra. ${ }^{14,15}$ Both biopolymers contained all five possible configurations, similar to that reported previously. ${ }^{14,15}$ Overall, the data show that cork suberin has longer polyester chains, due to a greater degree of esterification (viz., higher glycerol content, and more acylglycerol and primary esters), compared to potato suberin (Table 1).

Since the esterification level of each polyester type was distinctively evaluated, i.e., levels of secondary esters in cutin and acylglycerol esters in suberin, ${ }^{31} \mathrm{P}$ NMR was performed to systematically quantify specific free chemical groups in all samples. This methodology was initially proposed for the quantitative analysis of lignocellulosic polymers and involves the selective labeling of free $\mathrm{OH}$ groups. ${ }^{22}$ The presence of free acids and $\mathrm{OH}$ aliphatics could be detected in all samples. This confirms the presence of free acids in pepper cutin, despite the lack of a corresponding signal in both the ${ }^{1} \mathrm{H}$ and HSQC NMR spectra for this sample. Cutin samples have 5-10 times lower amounts of free acids compared to suberin samples. While tomato cutin and both suberin samples have similar $\mathrm{OH}$ aliphatic content, pepper cutin has a striking 7- to 17 -fold lower amount of this component. Both suberin exhibit similar high levels of $\mathrm{OH}$ aromatics; functionalities are also found in pepper cutin but at very low amounts (Table 1).

Through the use of high-resolution NMR-based methods, the esterification level in cutin and suberin was quantified. The identification and quantification of mid-chain and end-chain chemical groups in these materials were achieved, reflecting their astonishing chemical variability: each material reflects a particular polyester chemistry. A schematic representation of these findings, along with the relative abundance of selected components for each polyester, is depicted in Figure 3.

Cutin and Suberin Polymeric Arrangement Significantly Impacts Their Physicochemical Properties. The composition of a plant polyester dictates its structural organization and thus is responsible for its physicochemical properties. It is important to identify critical structural features of plant polyesters, including those at the molecular level, as these greatly influence the macrostructural arrangement and thus the physicochemical properties of these polymeric materials. To this end, DSC and X-ray scattering techniques, which provide information about the thermal profile and fundamental structural features of materials, respectively (Figure 4), have been employed in combination with NMR results (Table 1 and Figure 2) to establish relationships between chemical composition and structural properties.

The DSC thermograms acquired for both cutin and suberin samples demonstrate pronounced melting peak $(\mathrm{s})$ that indicate the presence of crystalline component(s) in these materials. Tomato cutin shows a higher enthalpy $\left(\Delta H=98.1 \mathrm{~J} \cdot \mathrm{g}^{-1}\right)$ compared to pepper cutin $\left(\Delta H=49.7 \mathrm{~J} \cdot \mathrm{g}^{-1}\right.$; Figure $\left.4 \mathrm{a}\right)$, suggesting that the former has a higher degree of crystallinity. 

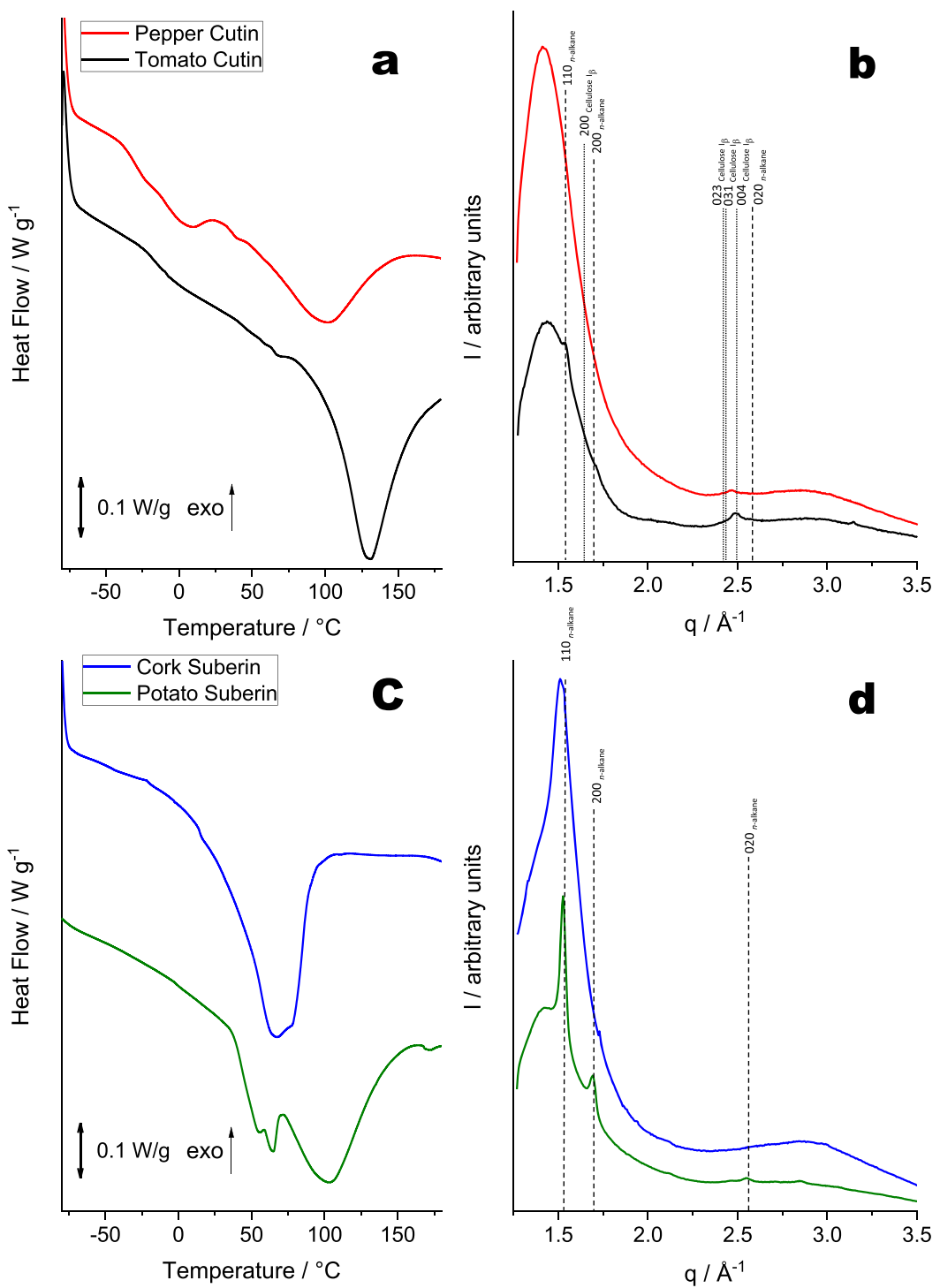

Figure 4. DSC thermograms (a, c) and WAXS patterns (b, d) collected for cutin and suberin samples isolated using cholinium hexanoate extraction for $2 \mathrm{~h}$. The vertical straight lines in the WAXS patterns indicate the position of diffraction peaks of cellulose (dotted lines) and crystallized $n$-alkane chains (dashed lines). Miller indexes assigned to the lines correspond to $\mathrm{I}_{\beta}$ cellulose (monoclinic space group $P 12_{1} 1$ ) and $n$-alkane chain packing (orthorhombic space group Pnma).

This is also consistent with the WAXS results (Figure $4 \mathrm{~b}$ ). The WAXS pattern obtained for the tomato cutin matches that reported previously for a similar material: ${ }^{16}$ a broad peak at $q \sim$ $1.41 \AA^{-1}$ and two weak diffraction peaks at $q \sim 1.52$ and $1.69 \AA^{-1}$ (Figure $4 \mathrm{~b}$, black curve) corresponding to $d$-spacings of 4.45 , 4.13, and 3.72 Å. Such a WAXS pattern is characteristic of a semicrystalline polymer with small crystalline domains. The broad peak $(d \sim 4.45 \AA)$ can be assigned to the interchain distance between acyl chains forming either an amorphous polymer structure ${ }^{35}$ or a hexagonal packing similar to rotator phases. ${ }^{36}$ The two weak diffraction peaks can be attributed to domains of acyl chains ordered in an orthorhombic crystal structure (space group Pnma, Miller indexes 110 and 200, respectively) usually formed by molecules that consist of alkanelike chains such as triacylglycerols $\left(\beta^{\prime} \text { phase }\right)^{37}$ or polyethylene. ${ }^{38,39}$ None of these diffraction peaks (that indicate the formation of well-ordered crystalline moieties in tomato cutin) could be observed in the WAXS patterns for pepper cutin (Figure $4 \mathrm{~b}$, red curve). The more amorphous nature of pepper cutin is possibly due to the abundance of secondary esters and epoxide groups. In tomato cutin, secondary esters are present at a lower amount and epoxide groups are entirely absent (Table 1).

The orthorhombic crystal structure observed in tomato cutin is consistent with its higher melting temperature $\left(123{ }^{\circ} \mathrm{C}\right)$ compared with that of pepper cutin $\left(100{ }^{\circ} \mathrm{C}\right)$ (Figure $\left.4 \mathrm{a}\right)$. The lower esterification level of the acyl chains in tomato cutin, compared with pepper cutin, possibly facilitates the formation of an orthorhombic unit cell. Such a unit cell is thermodynamically more stable than a rotator phase that is likely to be formed by distorted (esterified) alkane chains packed in a hexagonal array. ${ }^{36}$ Collectively, the acquired data strongly suggest that both the esterification level (mainly related to the presence of secondary esters in cutin) and the presence of mid-chain epoxide groups are the key features that control packing of the aliphatic chains; increasing the esterification level and abundance of midchain epoxides reduces the crystallinity and melting temperature of cutin. 
Finally, the diffraction peak at $q \sim 2.46 \AA^{-1}$, observed for both cutin samples (Figure $4 \mathrm{~b}$ ), cannot be related to the acyl-chain crystalline structure. Its position is significantly shifted from a possible (020) peak of the orthorhombic structure expected at $q$ $=2.55 \AA^{-1}$ (Figure $4 \mathrm{~b}$ ). This peak is likely to be associated with crystalline cellulose and can be assigned to a (004) reflection of monoclinic $\mathrm{I}_{\beta}$ cellulose (space group P12 1 ). ${ }^{40}$ Notably, the most intense (200) diffraction peak of the $\mathrm{I}_{\beta}$ cellulose expected at $q=1.63 \AA^{-1}$ is not visible because of an overlap with an intense broad peak that corresponds to the amorphous polymer structure. Crystalline cellulose usually coexists with amorphous cellulose, ${ }^{40}$ but it would be difficult to identify the amorphous cellulose component, with its expected maximum peak intensity at $q=1.52 \AA^{-1}$, from the broad diffuse peak observed in cutin's WAXS patterns. Using specific antibodies for crystalline cellulose, comparable semiquantitative levels were estimated for both samples (Figure S17). The amount of hydrolyzable carbohydrates $(<0.05 \%)$ is higher in tomato cutin than in pepper cutin (Table S2).

The thermal characterization of suberin samples revealed that a much higher enthalpy was needed to melt the crystals in potato suberin $\left(\Delta H=117.6 \mathrm{~J} \cdot \mathrm{g}^{-1}\right)$ compared to cork suberin $(\Delta H=81$

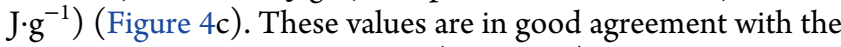
corresponding WAXS patterns (Figure 4d). The cork suberin pattern shows a broad peak between $q=1.35$ and $1.6 \AA^{-1}$, similar to cutin (Figure $4 \mathrm{~b}$ ), overlapping with a relatively sharp diffraction peak at $q \sim 1.5 \AA^{-1}$ (Figure $4 d$, blue curve). This pattern is characteristic of an amorphous polymer with a significant amount of crystalline component indicated by the diffraction peak at $q \sim 1.5 \AA^{-1}(d \sim 4.2 \AA)$, which could correspond to a (100) reflection of a hexagonal rotator phase structure formed by acyl-chain packing. The potato suberin WAXS pattern shows a broad peak similar to that observed for the cork suberin and also exhibits three additional pronounced peaks at $q=1.52,1.69$, and $2.55 \AA^{-1}$ that are characteristic of crystalline moieties corresponding to an orthorhombic structure (Figure 4d, green curve). In contrast to cutin, WAXS patterns for suberin showed no signs of crystalline cellulose. Very low amounts of hydrolyzable carbohydrates could be detected in these samples (Table S2).

The calorimetric analysis of both suberin samples revealed a thermal profile that is strikingly distinct from cutin, specifically the presence of more than one melting point. In particular, potato suberin has three melting temperatures $(56,65$, and 103 ${ }^{\circ} \mathrm{C}$ ), while cork suberin exhibits two melting temperatures (67 and $78{ }^{\circ} \mathrm{C}$; Figure $4 \mathrm{c}$ ). The more organized crystalline structure of potato suberin naturally results in both higher enthalpy and melting temperature compared to cork suberin. As concluded for cutin, the formation of crystalline structures is influenced by the esterification level, which in suberin is mainly dictated by the presence of both acylglycerol esters and epoxide groups. Hence, in cork suberin, the presence of epoxide groups hinders the packing of the aliphatic chains, rendering a less organized polymeric structure. Notably, the orthorhombic structure observed in the potato suberin WAXS pattern matches that observed in the tomato cutin (Figure $4 \mathrm{~b}$, black curve), showing an unexpected similarity between these two distinct types of plant polyesters at the nanoscale. Both potato suberin and tomato cutin have lower esterification levels than their counterparts, but the former has more orthorhombic crystalline moieties. This shows that the packing of the aliphatic chains is eased by the presence of acylglycerol linkages present in suberin but not by the secondary esters present in cutin. This finding is also consistent with previous studies. ${ }^{37}$

Overall, the acquired data show that the exploitation of different plant sources, containing plant polyesters with distinct chemistries, can be used to tune the physical properties of extracted materials. Considering the range of physicochemical properties exhibited by different plant polyesters, or by polyesters from different sources, it would be desirable to continue to use tomato cutin as a model with which to further analyze how specific structural alterations influence physicochemical properties.

Genetic Modifications Allow the Fine-Tuning of Cutin Physicochemical Properties. The physicochemical properties of polyesters from a defined genotype, Micro-Tom cultivar, and from two of its well-characterized mutants were analyzed. The selected mutants were gpat6 (GLYCEROL-3-PHOSPHATE ACYLTRANSFERASE gene) and cus1 (CUTIN SYNTHASE gene $)^{32,41,42}$ that exhibit altered cutin composition and altered degrees of intrachain branching. In the gpat6 mutant (formerly named cutin-deficient mutant $c u d 1),{ }^{32}$ the synthesis of the major cutin precursor is hindered, resulting in a thinner cuticle with decreased levels of cutin but enriched in fatty acids. ${ }^{41}$ In the cus 1 mutant, cutin polymerization is impaired ${ }^{43,44}$ and the presence of secondary esters is greatly reduced. ${ }^{32}$ The cutin samples were purified from tomato peels using the established ionic liquid method $^{16}$ and analyzed using the same procedures used for other samples in this study. SEM revealed that all cutin samples extracted from Micro-Tom tomato peels (Figure S18) showed similar morphologies to those of both tomato and pepper cutin (Figure 1), as well as to those previously reported by us for cutin from the same genotype. ${ }^{16}$ Our main goal was to analyze if specific alterations in cutin biosynthesis would lead to predictable alterations in the physicochemical properties of the resulting polymers. This constitutes an unmatched approach to confirm the importance of cutin esterification levels and how they relate to its thermal resistance and crystallinity.

The assignment of the NMR signals was attained through a combination of $2 \mathrm{D}$ spectra $\left({ }^{1} \mathrm{H}-{ }^{1} \mathrm{H}\right.$ COSY, ${ }^{1} \mathrm{H}-{ }^{13} \mathrm{C}$ HSQC, and ${ }^{1} \mathrm{H}-{ }^{13} \mathrm{C}$ HMBC; Figures $\left.\mathrm{S} 19-\mathrm{S} 27\right)$ and is in good agreement with a previous report. ${ }^{16}$ The magnification of the HSQC regions corresponding to aliphatics and $\mathrm{CH} / \mathrm{CH} 2-\mathrm{X}$ aliphatics for all cutin samples (Figure S28) revealed that both cutins extracted from the mutant strains are enriched in $\mathrm{CH}_{3}$ groups compared to cutin from the wild-type strain. This is consistent with previous studies. ${ }^{16,32,41}$ All of the crucial quantifications required for the analysis of the polymeric structural organization are summarized in Table 2. As anticipated, the analyzed cutin samples exhibit great differences in the degree of esterification and in the content of $\mathrm{OH}$ aliphatics. The cutin extracted from the wild-type Micro-Tom cultivar is more esterified than that of both mutants; cutin from the gpat6 mutant is the least esterified. The trend observed for the amount of secondary esters (normalized values) is wild-type $>$ gpat6 $>$ cus1. The trend for the amount of $\mathrm{OH}$ aliphatics, calculated by ${ }^{31} \mathrm{P} \mathrm{NMR}$, is cus $1>$ gpat6 > wild-type. The observed increase in the content of free $\mathrm{OH}$ aliphatics correlates with a decrease in the secondary ester content. This observation is also in general agreement with previously published data on these mutants that was obtained using an independent approach (detection of benzyl etherified $\mathrm{OH}$ groups in cutin hydrolysates). ${ }^{32}$ The amount of free $\mathrm{OH}$ groups detected by ${ }^{31} \mathrm{P}$ NMR may include some free $\mathrm{OH}$ groups from cuticular polysaccharides (regardless of their very low abundance; Table S2). 
Table 2. Chemical Characterization of Cutin Samples from Micro-Tom Cultivars ${ }^{a}$

\begin{tabular}{|c|c|c|c|c|}
\hline & & \multicolumn{3}{|c|}{$\begin{array}{l}\text { purified cutin from Micro-Tom } \\
\text { tomatoes peels }\end{array}$} \\
\hline \multicolumn{2}{|c|}{ NMR } & wild-type & cus1 & gpat6 \\
\hline \multicolumn{2}{|l|}{ primary esters $^{b}$} & $66[6.6]$ & $78[6.24]$ & $25[0.75]$ \\
\hline \multicolumn{2}{|l|}{ secondary esters $b$} & $34[3.4]$ & $22[1.76]$ & $75[2.25]$ \\
\hline \multicolumn{2}{|l|}{ total esters ${ }^{c}$} & 10 & 8 & 3 \\
\hline \multicolumn{2}{|l|}{ free acids ${ }^{d}$} & 0.14 & n.d. & n.d. \\
\hline \multirow{3}{*}{$\begin{array}{l}{ }^{31} \mathrm{P} \text { quantification } \\
\left(\mathrm{mmol} \cdot \mathrm{g}^{-1}\right)\end{array}$} & free acids & 0.065 & 0.067 & 0.029 \\
\hline & $\mathrm{OH}$ aliphatics & 2.467 & 7.82 & 7.609 \\
\hline & $\mathrm{OH}$ aromatics & 0.168 & n.d. & n.d. \\
\hline
\end{tabular}

${ }^{a}$ NMR quantification of the main functional groups in the cutin samples derived from Micro-Tom tomato peel-wild-type, cus1, and gpat6-isolated using cholinium hexanoate extraction for $2 \mathrm{~h}$. ${ }^{b}$ Relative abundances of primary and secondary esters present in cutin calculated through integration of signals in the ${ }^{1} \mathrm{H}$ NMR spectra. Values in brackets show the normalized values using the amount of total esters in each sample. ${ }^{c}$ Integration of the total ester signal was normalized with benzene for comparison between samples. ${ }^{d}$ Content of free acids relative to the total area of ${ }^{1} \mathrm{H}$ spectrum.

Additionally, free acids are less abundant in the gpat 6 cutin and $\mathrm{OH}$ aromatics are only detected in the wild-type cutin. Here, the structural features (total, primary, and secondary esters; Table 2) were precisely assigned and constitute absolute values. This differs from a previous study on similar cutin materials where their relative abundances were qualitatively inferred. ${ }^{16}$ The ability to systematically compare the absolute quantities of structural features associated with mutations that affect cutin biosynthesis is a powerful methodology, which deserves a more focused analysis in the near future.

The DSC thermograms of all Micro-Tom cutin samples (Figure 5a) show considerably higher melting temperatures for cutin from both mutants (cus1 $169^{\circ} \mathrm{C}$, and gpat6 $175{ }^{\circ} \mathrm{C}$ ) compared to the wild type $\left(131^{\circ} \mathrm{C}\right)$. The thermal resistance of the wild-type cutin is close to that observed for tomato cutin purified from commercial tomatoes $\left(123{ }^{\circ} \mathrm{C}\right.$, Figure $\left.4 \mathrm{a}\right)$, reflecting the impact of the plant raw material. The enthalpy required to melt the crystalline moieties present in the polymeric materials shows a similar trend: cus 1 and gpat6 cutin samples display much higher enthalpy (cus1 $159 \mathrm{~J} \cdot \mathrm{g}^{-1}$, and gpat6 $124 \mathrm{~J}$. $\left.\mathrm{g}^{-1}\right)$ compared to the wild type $\left(82 \mathrm{~J} \cdot \mathrm{g}^{-1}\right)$. The WAXS patterns of the Micro-Tom tomato cutin samples (Figure $5 b$ ) are similar to those obtained for the commercial tomato cutin (Figure $4 \mathrm{~b}$ ): a broad band near $q=1.44 \AA^{-1}$ and three weak diffraction peaks at $q \sim 1.52,1.69$, and $2.46 \AA^{-1}$ (Figure 5b). The peaks related to the orthorhombic structure of the crystals are, however, more pronounced in the cutin extracted from both mutants compared to that of the wild type. This reflects the higher degree of crystallinity found in the cutin extracted from the mutants. The third diffraction peak at $q \sim 2.46 \AA^{-1}$ observed for all MicroTom cutin samples should be related to the (004) reflection of monoclinic $\mathrm{I}_{\beta}$ cellulose (space group $P 12_{1} 1$ ) (Figure $5 \mathrm{~b}$ ), in analogy to both commercial tomato and pepper cutin (Figure $4 \mathrm{~b})$. The presence of crystalline cellulose could be confirmed through immunoblotting (Figure S17). The estimated, semiquantitative values make it evident that a lower proportion of microcrystalline cellulose is present in cutin from wild-type plants compared to either mutant. The results obtained are consistent with WAXS results showing that the crystalline cellulose diffraction peak of the wild-type cutin is significantly less intense in comparison with that from the mutant cutins (Figure $5 b$ ). Interestingly, the cutin samples extracted from the peel of wild-type cultivars, the commercial cultivar, and the miniaturized Micro-Tom tomatoes showed almost identical levels of microcrystalline cellulose (Figure S17) and of total hydrolyzable carbohydrates (Table S2). The last are also comparable in either cutin extracted from the mutants. These results suggest that the higher thermal resistance of the cutin extracted from either mutant may include the contribution from cuticular polysaccharides (regardless of their very low abundance; Table S2). The presence of cuticular polysaccharides in either cutin sample is consistent with data recently reported for tomato cutin extracted using the conventional enzymatic-based approach. ${ }^{30}$ This result adds support to
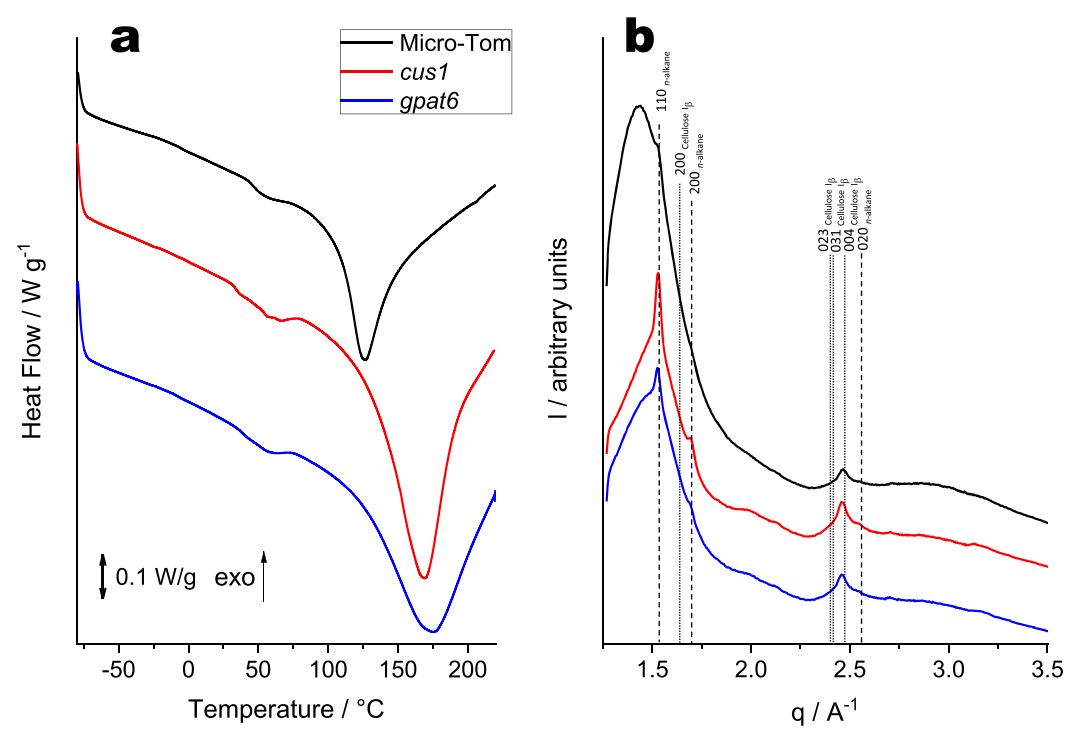

Figure 5. DSC thermograms (a) and WAXS patterns (b) collected for cutin samples extracted from Micro-Tom tomato peel-wild-type, cus1, and gpat6-isolated using cholinium hexanoate extraction for $2 \mathrm{~h}$. The vertical straight lines in WAXS patterns indicate the position of diffraction peaks of cellulose (dotted lines) and crystallized $n$-alkane chains (dashed lines). Miller indexes assigned to the lines correspond to $\mathrm{I}_{\beta}$ cellulose (monoclinic space group $P 12{ }_{1} 1$ ) and $n$-alkane chain packing (orthorhombic space group Pnma). 
previous data that suggested the existence of crosstalk between lipid and polysaccharide deposition in these mutant plant cuticles; $^{30,41}$ either mutation would affect both the cutin polymer and the cuticular polysaccharides.

Finally, the low crystallinity of the wild-type cutin is likely related to its high degree of esterification along with the high amount of secondary esters. The cus1 mutant has a more crystalline cutin due to the low abundance of secondary esters (normalized values) and high $\mathrm{OH}$ aliphatics content. These modifications allow the polymeric chains to pack into an orthorhombic structure. Although the amount of secondary esters in cutin extracted from the gpat6 mutant has an intermediate value (i.e., higher than cus1 cutin but lower than the wild type), it is more crystalline than the wild-type cutin. We hypothesize that the high content of unbound $\mathrm{OH}$ aliphatics in conjunction with the shorter polymeric chain lengths (inferred by the low amount of primary esters) facilitates the organization of the polymeric chains into a more crystalline material. Additionally, the enrichment of fatty acids in either mutant cutin may also play a crucial role in the crystallization of the polymeric chains.

\section{CONCLUSIONS}

Plant polyesters constitute multifunctional polymers that can be isolated preserving high esterification levels. Through a systematic analysis of the chemistry, structure, thermal resistance, and crystallinity of distinctive plant polyesters, we have identified the most critical "natural" structural features that influence their material properties. Specifically, quantitative NMR, DSC, and X-ray scattering data were collected and their integration defines the Rules Of Thumb For The Quantification Of The Structure-Property Relationships Of Plant Polyesters. These rules are outlined below:

1. Despite the amorphous nature of the polymeric materials analyzed here, the degree of esterification dictates the packing of the aliphatic chains (also influenced by their length) and hence polymer crystallinity (and the resultant crystal structure) and melting temperature.

2. A low degree of esterification favors the formation of crystalline regions, yet the packing of the aliphatic chains is easier to accomplish in the presence of acylglycerol bonds compared to secondary esters.

3. The packing of the aliphatic chains is greatly influenced by the presence of mid-chain epoxide groups.

4. Increased amounts of unbound $\mathrm{OH}$ aliphatic groups and smaller chain lengths (inferred from the amount of primary esters) facilitate the organization of the polymeric chains.

5. A low degree of esterification of the acyl chains in the polyester favors the formation of alkane microcrystals with an orthorhombic structure.

How the molecular weight of the polymer correlates with the physicochemical properties remains unanswered because their broad insolubility in organic solvents hinders the development of a method with which to analyze both polyester types. Further characterization of their mechanical properties (in addition to their molecular weight) will be essential to refine these proposed rules, with the eventual aim of developing a user-friendly computing toolbox.

Finally, it is worth reinforcing the broad applicability of the quantitative NMR workflow developed here for the collection of thorough quantitative molecular structure data on diverse plant polyesters. This can be applied in materials sciences, specifically for the design of polyester materials, supporting the selection of suitable plant sources and/or downstream processes (e.g., control of esterification level/type). The workflow also constitutes an essential tool for the structural characterization of polyesters from model plant genotypes and may support the identification of cultivars carrying features that may be correlated with a higher resistance to abiotic pressures (e.g., climate change).

To conclude, we have deduced the Rules Of Thumb For The Quantification Of The Structure-Property Relationships Of Plant Polyesters following a systematic characterization of the structure, chemistry, thermal resistance, and crystallinity of two polyesters types, in both cases greatly preserving the natural polymeric backbone. Unexpectedly, similar crystal structures were observed in distinct polyester types (potato suberin and tomato cutin) and highly thermally resistant cutin was found in both Micro-Tom mutants. The future holds promise; we have started to learn how to tune specific properties of plant polyesters through genetic manipulation or through the selection of different source materials, supporting the development of materials for specific applications in the future.

\section{ASSOCIATED CONTENT}

\section{Supporting Information}

The Supporting Information is available free of charge at https://pubs.acs.org/doi/10.1021/acssuschemeng.1c04733.

Schematic illustration of polymer isolation; GC-MS chromatograms; NMR spectra and assignment table; SEM micrographs; schematics of the extraction process; representative GC-MS chromatograms of the model compound sucrose monolaurate; elemental analysis of tomato cutin, pepper cutin, cork suberin, and potato suberin; total carbohydrate quantification of cutin and suberin samples; quantification of crystalline cellulose concentration with ImageJ software, of cutin from tomato, pepper, Micro-tom wild type, and mutants purified with cholinium hexanoate; and wide-ranging NMR spectral characterization of Micro-tom cutins (PDF)

\section{AUTHOR INFORMATION}

\section{Corresponding Author}

Cristina Silva Pereira - Instituto de Tecnologia Química e Biológica António Xavier, Universidade Nova de Lisboa

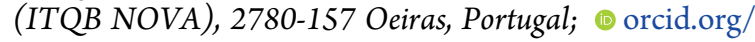
0000-0002-6750-1593; Email: spereira@itqb.unl.pt

\section{Authors}

Artur Bento - Instituto de Tecnologia Química e Biológica António Xavier, Universidade Nova de Lisboa (ITQB NOVA), 2780-157 Oeiras, Portugal; @ orcid.org/00000003-4097-1613

Carlos J. S. Moreira - Instituto de Tecnologia Química e Biológica António Xavier, Universidade Nova de Lisboa (ITQB NOVA), 2780-157 Oeiras, Portugal; (i) orcid.org/ 0000-0002-7708-2505

Vanessa G. Correia - Instituto de Tecnologia Química e Biológica António Xavier, Universidade Nova de Lisboa (ITQB NOVA), 2780-157 Oeiras, Portugal; 이잉.org/ 0000-0002-7357-7199

Rita Escórcio - Instituto de Tecnologia Química e Biológica António Xavier, Universidade Nova de Lisboa (ITQB 
NOVA), 2780-157 Oeiras, Portugal; 이잉.org/00000003-3473-3686

Rúben Rodrigues - Instituto de Tecnologia Química e Biológica António Xavier, Universidade Nova de Lisboa (ITQB NOVA), 2780-157 Oeiras, Portugal

Ana S. Tomé - Instituto de Tecnologia Química e Biológica António Xavier, Universidade Nova de Lisboa (ITQB NOVA), 2780-157 Oeiras, Portugal

Nathalie Geneix - INRAE, UR BIA 1268, 44316 Nantes, France

Johann Petit - UMR 1332 BFP, INRAE, Univ. Bordeaux, F33140 Villenave d'Ornon, France

Bénédicte Bakan - INRAE, UR BIA 1268, 44316 Nantes, France

Christophe Rothan - UMR 1332 BFP, INRAE, Univ. Bordeaux, F-33140 Villenave d'Ornon, France; 이이.org/ 0000-0002-6831-2823

Oleksandr O. Mykhaylyk - Soft Matter Analytical Laboratory, Dainton Building, Department of Chemistry, The University of

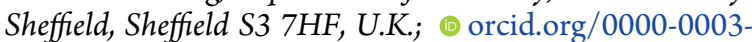
4110-8328

Complete contact information is available at:

https://pubs.acs.org/10.1021/acssuschemeng.1c04733

\section{Author Contributions}

C.J.S.M. and V.G.C. contributed equally. C.S.P and O.O.M. contributed to conceptualization. C.S.P. and O.O.M. were responsible for funding acquisition. Project administration and supervision was done by C.S.P. C.S.P., O.O.M., B.B., and C.R. contributed to resources. All authors contributed to investigation and methodology. Formal analysis was conducted by A.B., C.J.S.M., V.G.C., and O.O.M. Validation, visualization, and writing-original draft were performed by A.B., C.J.S.M., and V.G.C. All authors contributed to writing-review and editing.

\section{Notes}

The authors declare no competing financial interest.

\section{ACKNOWLEDGMENTS}

The authors acknowledge funding from the European Research Council through grant ERC 2014-CoG-647928, from the European Union's Horizon 2020 research and innovation program within the project 713475-FLIPT-H2020-FETOPEN-2014-2015, and from Fundação para a Ciência e Tecnologia (FCT) by Project MOSTMICRO ITQB with refs UIDB/04612/2020 and UIDP/04612/2020. The NMR data were acquired at CERMAX, ITQB NOVA, Oeiras, Portugal, with equipment funded by FCT. C.J.S.M. is grateful to Aralab, Portugal, for the PhD contract 06/PlantsLife/2017. O.O.M. thanks EPSRC for a capital equipment grant to purchase the Xenocs/Excillum SAXS/WAXS laboratory beamline (EP/ M028437/1). The authors are thankful to Pedro Lamosa and Maria C. Leitão (ITQB NOVA) for support with the NMR and chromatographic analyses, respectively, and to James Yates (ITQB NOVA) for proofreading the manuscript.

\section{REFERENCES}

(1) Heredia-Guerrero, J. A.; Athanassiou, A. Editorial: NonPolysaccharide Plant Polymeric Materials. Front. Mater. 2016, 3, No. 15.

(2) Siddique, R.; Khatib, J.; Kaur, I. Use of Recycled Plastic in Concrete: A Review. Waste Manag. 2008, 28, 1835-1852.
(3) Galloway, T. S.; Cole, M.; Lewis, C. Interactions of Microplastic Debris throughout the Marine Ecosystem. Nat. Ecol. Evol. 2017, 1, No. 0116.

(4) Matthews, C.; Moran, F.; Jaiswal, A. K. A Review on European Union's Strategy for Plastics in a Circular Economy and Its Impact on Food Safety. J. Clean. Prod. 2021, 283, No. 125263.

(5) Philippe, G.; Sørensen, I.; Jiao, C.; Sun, X.; Fei, Z.; Domozych, D. S.; Rose, J. K. Cutin and Suberin: Assembly and Origins of Specialized Lipidic Cell Wall Scaffolds. Curr. Opin. Plant Biol. 2020, 55, 11-20.

(6) Vishwanath, S. J.; Delude, C.; Domergue, F.; Rowland, O. Suberin: Biosynthesis, Regulation, and Polymer Assembly of a Protective Extracellular Barrier. Plant Cell Rep. 2015, 34, 573-586.

(7) Martin, L. B. B.; Rose, J. K. C. There's More than One Way to Skin a Fruit: Formation and Functions of Fruit Cuticles. J. Exp. Bot. 2013, 65, 4639-4651.

(8) Schreiber, L. Transport Barriers Made of Cutin, Suberin and Associated Waxes. Trends in Plant Science; Elsevier, 2010; pp 546-553.

(9) Nawrath, C. The Biopolymers Cutin and Suberin. Arabidopsis Book 2002, 1, No. e0021.

(10) Sousa, A. F.; Gandini, A.; Silvestre, A. J. D.; Pascoal Neto, C. Synthesis and Characterization of Novel Biopolyesters from Suberin and Model Comonomers. ChemSusChem 2008, 1, 1020-1025.

(11) Chatterjee, S.; Sarkar, S.; Oktawiec, J.; Mao, Z.; Niitsoo, O.; Stark, R. E. Isolation and Biophysical Study of Fruit Cuticles. J. Vis. Exp. 2012, No. e3529.

(12) Graça, J.; Lamosa, P. Linear and Branched Poly $(\omega$-Hydroxyacid) Esters in Plant Cutins. J. Agric. Food Chem. 2010, 58, 9666-9674.

(13) Ferreira, R.; Garcia, H.; Sousa, A. F.; Guerreiro, M.; Duarte, F. J. S.; Freire, C. S. R.; Calhorda, M. J.; Silvestre, A. J. D.; Kunz, W.; Rebelo, L. P. N.; et al. Unveiling the Dual Role of the Cholinium Hexanoate Ionic Liquid as Solvent and Catalyst in Suberin Depolymerisation. RSC Adv. 2014, 4, 2993-3002.

(14) Correia, V. G.; Bento, A.; Pais, J.; Rodrigues, R.; Haliński, P.; Frydrych, M.; Greenhalgh, A.; Stepnowski, P.; Vollrath, F.; King, A. W. T.; et al. The Molecular Structure and Multifunctionality of the Cryptic Plant Polymer Suberin. Mater. Today Bio 2020, 5, No. 100039.

(15) Rodrigues, R.; Palma, S. I. C. J.; G Correia, V.; Padrão, I.; Pais, J.; Banza, M.; Alves, C.; Deuermeier, J.; Martins, C.; Costa, H. M. A.; et al. Sustainable Plant Polyesters as Substrates for Optical Gas Sensors. Mater. Today Bio 2020, 8, No. 100083.

(16) Moreira, C. J. S.; Bento, A.; Pais, J.; Petit, J.; Escórcio, R.; Correia, V. G.; Pinheiro, A.; Haliński, P.; Mykhaylyk, O. O.; Rothan, C.; et al. An Ionic Liquid Extraction That Preserves the Molecular Structure of Cutin Shown by Nuclear Magnetic Resonance. Plant Physiol. 2020, 184, 592-606.

(17) Benítez, J. J.; Castillo, P. M.; del Río, J. C.; León-Camacho, M.; Domínguez, E.; Heredia, A.; Guzmán-Puyol, S.; Athanassiou, A.; Heredia-Guerrero, J. A. Valorization of Tomato Processing ByProducts: Fatty Acid Extraction and Production of Bio-Based Materials. Materials 2018, 11, No. 2211.

(18) Marc, M.; Risani, R.; Desnoes, E.; Falourd, X.; Pontoire, B.; Rodrigues, R.; Escórcio, R.; Batista, A. P.; Valentin, R.; Gontard, N.; et al. Bioinspired Co-Polyesters of Hydroxy-Fatty Acids Extracted from Tomato Peel Agro-Wastes and Glycerol with Tunable Mechanical, Thermal and Barrier Properties. Ind. Crops Prod. 2021, 170, No. 113718.

(19) Bedoić, R.; Ćosić, B.; Duić, N. Technical Potential and Geographic Distribution of Agricultural Residues, Co-Products and by-Products in the European Union. Sci. Total Environ. 2019, 686, 568-579.

(20) Just, D.; Garcia, V.; Fernandez, L.; Bres, C.; Mauxion, J.-P.; Petit, J.; Jorly, J.; Assali, J.; Bournonville, C.; Ferrand, C.; et al. Micro-Tom Mutants for Functional Analysis of Target Genes and Discovery of New Alleles in Tomato. Plant Biotechnol. 2013, 30, 225-231.

(21) Petkovic, M.; Ferguson, J. L.; Gunaratne, H. Q. N.; Ferreira, R.; Leitão, M. C.; Seddon, K. R.; Rebelo, L. P. N.; Pereira, C. S. Novel Biocompatible Cholinium-Based Ionic Liquids-Toxicity and Biodegradability. Green Chem. 2010, 12, 643. 
(22) Meng, X.; Crestini, C.; Ben, H.; Hao, N.; Pu, Y.; Ragauskas, A. J.; Argyropoulos, D. S. Determination of Hydroxyl Groups in Biorefinery Resources via Quantitative 31P NMR Spectroscopy. Nat. Protoc. 2019, $14,2627-2647$.

(23) Blake, A. W.; McCartney, L.; Flint, J. E.; Bolam, D. N.; Boraston, A. B.; Gilbert, H. J.; Knox, J. P. Understanding the Biological Rationale for the Diversity of Cellulose-Directed Carbohydrate-Binding Modules in Prokaryotic Enzymes. J. Biol. Chem. 2006, 281, 29321-29329.

(24) Mattinen, M. L.; Filpponen, I.; Järvinen, R.; Li, B.; Kallio, H.; Lektinen, P.; Argyropoulos, D. Structure of the Polyphenolic Component of Suberin Isolated from Potato (Solanum tuberosum War. Nikola). J. Agric. Food Chem. 2009, 57, 9747-9753.

(25) Liang, S.; McDonald, A. G. Chemical and Thermal Characterization of Potato Peel Waste and Its Fermentation Residue as Potential Resources for Biofuel and Bioproducts Production. J. Agric. Food Chem. 2014, 62, 8421-8429.

(26) Johnson, E. J.; Dorot, O.; Liu, J.; Chefetz, B.; Xing, B. Spectroscopic Characterization of Aliphatic Moieties in Four Plant Cuticles. Commun. Soil Sci. Plant Anal. 2007, 38, 2461-2478.

(27) Chen, B. 1.; Li, Y. g. Sorption of 1-Naphthol by Plant Cuticular Fractions. J. Environ. Sci. 2007, 19, 1214-1220.

(28) Hartman, B. E.; Hatcher, P. G. Hydrothermal Liquefaction of Isolated Cuticle of Agave Americana and Capsicum Annuum: Chemical Characterization of Petroleum-like Products. Fuel 2015, 156, 225-233.

(29) Xin, A.; Fry, S. C. Cutin:Xyloglucan Transacylase (CXT) Activity Covalently Links Cutin to a Plant Cell-Wall Polysaccharide. J. Plant Physiol. 2021, 262, No. 153446.

(30) Philippe, G.; Geneix, N.; Petit, J.; Guillon, F.; Sandt, C.; Rothan, C.; Lahaye, M.; Marion, D.; Bakan, B. Assembly of Tomato Fruit Cuticles: A Cross-talk between the Cutin Polyester and Cell Wall Polysaccharides. New Phytol. 2020, 226, 809-822.

(31) Deshmukh, A. P.; Simpson, A. J.; Hatcher, P. G. Evidence for Cross-Linking in Tomato Cutin Using HR-MAS NMR Spectroscopy. Phytochemistry 2003, 64, 1163-1170.

(32) Philippe, G.; Gaillard, C.; Petit, J.; Geneix, N.; Dalgalarrondo, M.; Bres, C.; Mauxion, J.-P.; Franke, R.; Rothan, C.; Schreiber, L.; et al. Ester Cross-Link Profiling of the Cutin Polymer of Wild-Type and Cutin Synthase Tomato Mutants Highlights Different Mechanisms of Polymerization. Plant Physiol. 2016, 170, 807-820.

(33) Fich, E. A.; Segerson, N. A.; Rose, J. K. C. The Plant Polyester Cutin: Biosynthesis, Structure, and Biological Roles. Annu. Rev. Plant Biol. 2016, 67, 207-233.

(34) Graça, J.; Santos, S. Glycerol-Derived Ester Oligomers from Cork Suberin. Chem. Phys. Lipids 2006, 144, 96-107.

(35) Heredia-Guerrero, J. A.; Domínguez, E.; Luna, M.; Benítez, J. J.; Heredia, A. Structural Characterization of Polyhydroxy Fatty Acid Nanoparticles Related to Plant Lipid Biopolyesters. Chem. Phys. Lipids 2010, 163, 329-333.

(36) Small, D. M. Lateral Chain Packing in Lipids and Membranes. J. Lipid Res. 1984, 25, 1490-1500.

(37) Mykhaylyk, O. O.; Smith, K. W.; Martin, C. M.; Ryan, A. J. Structural Models of Metastable Phases Occurring during the Crystallization Process of Saturated/Unsaturated Triacylglycerols. J. Appl. Crystallogr. 2007, 40, s297-s302.

(38) Southern, J. H.; Weeks, N.; Porter, R. S.; Crystal, R. G. Unique Polyethylene Morphologies Produced under Extrusion Conditions. Makromol. Chem. 1972, 162, 19-30.

(39) Bunn, C. W. The Crystal Structure of Ethylene. Trans. Faraday Soc. 1944, 40, 23-25.

(40) Rongpipi, S.; Ye, D.; Gomez, E. D.; Gomez, E. W. Progress and Opportunities in the Characterization of Cellulose - an Important Regulator of Cell Wall Growth and Mechanics. In Frontiers in Plant Science; Frontiers Media S.A., 2019; 1894.

(41) Petit, J.; Bres, C.; Mauxion, J. P.; Tai, F. W. J.; Martin, L. B. B.; Fich, E. A.; Joubès, J.; Rose, J. K. C.; Domergue, F.; Rothan, C. The Glycerol-3-Phosphate Acyltransferase GPAT6 from Tomato Plays a Central Role in Fruit Cutin Biosynthesis. Plant Physiol. 2016, 171, 894913.
(42) Petit, J.; Bres, C.; Mauxion, J.-P.; Bakan, B.; Rothan, C. Breeding for Cuticle-Associated Traits in Crop Species: Traits, Targets, and Strategies. J. Exp. Bot. 2017, 68, 5369-5387.

(43) Girard, A.-L.; Mounet, F.; Lemaire-Chamley, M.; Gaillard, C.; Elmorjani, K.; Vivancos, J.; Runavot, J.-L.; Quemener, B.; Petit, J.; Germain, V.; et al. Tomato GDSL1 Is Required for Cutin Deposition in the Fruit Cuticle. Plant Cell 2012, 24, 3119-3134.

(44) Yeats, T. H.; Martin, L. B. B.; Viart, H. M. F.; Isaacson, T.; He, Y.; Zhao, L.; Matas, A. J.; Buda, G. J.; Domozych, D. S.; Clausen, M. H.; et al. The Identification of Cutin Synthase: Formation of the Plant Polyester Cutin. Nat. Chem. Biol. 2012, 8, 609-611. 